\title{
On Vector-valued Inequalities of the Marcinkiewicz-Zygmund, Herz and Krivine Type
}

Dedicated to Professor Mischa CotLAR on the occasion of his 80th birthday

By J. GASCH of Caracas and L. MaLigranda of Luleå

(Received February 8, 1993)

\begin{abstract}
Any continuous linear operator $T: L^{p} \rightarrow L^{q}$ has a natural vector-valued extension $T_{E}: L^{p}\left(I_{n}^{r}\right) \rightarrow L^{q}\left(r_{n}^{r}\right)$ which is automatically continuous. Relations between the norms of these operators in the cases of $p=q$ and $r=2$ were considered by MARCINKIEWICZ-ZYGMUND [28], Herz [14] and KRIVINE [19]-[21]. In this paper we study systematically these relations and given some applications. It turns out that some known results can be proved in a simple way as a consequence of these developments.
\end{abstract}

\section{Introduction}

Exact relations between the norm of an operator and its complexification are very important in various branches of mathematics, in particular in interpolation theory. The fact that these norms are not always the same, even between finite-dimensional spaces, was observed already by M. RIESz [35] and G. O. THORIN [44]. Let $T$ be a bounded linear operator mapping from the real space $L^{p}(\mu)$ into another real space $L^{q}(v)$ with the norm $\|T\|$. Let $T_{C}$ be the complex extension of $T$, i.e.,

$$
T_{C}(f+i g):=T f+i T g,
$$

and let $\left\|T_{C}\right\|$ be the norm of $T_{C}$ from the complex extension $L_{C}^{p}(\mu)$ into the complex extension $L_{C}^{q}(v)$.

Of course $\|T\| \leq\left\|T_{C}\right\|$, but it is interesting to know the reverse estimate

$$
\left\|T_{C}\right\| \leq \gamma\|T\|
$$

for some $\gamma=\gamma\left(L^{p}(\mu), L^{q}(v)\right)$.

What does this inequality mean? We have

$$
\begin{aligned}
\left\|T_{C}(f+i g)\right\|_{q} & =\||T f+i T g|\|_{q}=\left\|\left(|T f|^{2}+|T g|^{2}\right)^{1 / 2}\right\|_{q} \\
& \leq\left\|T_{C}\right\|\|f+i g\|_{p} \leq \gamma\|T\|\||f+i g|\|_{p} \\
& =\gamma\|T\|\left\|\left(|f|^{2}+|g|^{2}\right)^{1 / 2}\right\|_{p} .
\end{aligned}
$$


Therefore $\gamma$ is the best constant in the inequality

$$
\left\|\left(|T f|^{2}+|T g|^{2}\right)^{1 / 2}\right\|_{q} \leq \gamma\|T\|\left\|\left(|f|^{2}+|g|^{2}\right)^{1 / 2}\right\|_{p}
$$

for all $f, g \in L^{p}(\mu)$ and any $T$.

We will consider the following generalizations of the complexification problem. For $1 \leq p, q, r \leq \infty, n=2,3, \ldots$ and for any linear bounded operator between real spaces $T: L^{p}(X, \mu) \rightarrow L^{q}(Y, v)$ let us consider the following expressions:

$$
\begin{aligned}
& K_{L^{p}(\mu), L^{q}(v)}^{(n)}(r)=\inf \left\{C \geq 1:\left\|\left(\sum_{k=1}^{n}\left|T f_{k}\right|^{r}\right)^{1 / r}\right\|_{q} \leq C\|T\|\left\|\left(\sum_{k=1}^{n}\left|f_{k}\right|^{r}\right)^{1 / r}\right\|_{p}\right. \\
& \text { for all } \left.f_{1}, f_{2}, \ldots, f_{n} \in L^{p}(X, \mu) \text { and any } T: L^{p}(X, \mu) \rightarrow L^{q}(Y, v)\right\}, \\
& K_{p, q}^{(n)}(r)=\sup \left\{K_{L^{p}(\mu), L^{q}(v)}^{(n)}(r): \mu, v, \sigma \text {-finite measures }\right\}
\end{aligned}
$$

and

$$
K_{p, q}(r)=\sup _{n \geq 2} K_{p, q}^{(n)}(r)
$$

We can also consider the constants $K_{p, q}^{(n)}(r)$ as well as the relationship between the norms of natural vector-valued extension $T_{E}$ and $T$. More precisely, we can consider the vector-valued operator (or, equivalently, the operator between mixed-norm spaces) $T_{E}: L^{p}\left(\mu . l_{n}^{r}\right) \rightarrow L^{q}\left(v, l_{n}^{r}\right)$ defined in the following way:

$$
T_{E}(f)=T_{E}\left(\left(f_{1}, f_{2}, \ldots, f_{n}\right)\right):=\left(T f_{1}, T f_{2}, \ldots, T f_{n}\right)
$$

for $f=\left(f_{1}, f_{2}, \ldots, f_{n}\right) \in L^{p}\left(\mu, l_{n}^{r}\right)$.

Then the supremum over the non-zero maps $T \in B\left(L^{p}(\mu), L^{q}(v)\right)$ of the norm of $T_{E}$ in $B\left(L^{p}\left(\mu, l_{n}^{r}\right), L^{q}\left(v, l_{n}^{r}\right)\right)$ over the norm of $T$ in $B\left(L^{p}(\mu), L^{q}(v)\right)$ is equal to $K_{p, q}^{(n)}(r)$, and thus

$$
K_{p, q}^{(n)}(r)=\sup \left\{\left\|T_{E}\right\|_{L^{p}\left(\mu, l_{n}\right) \rightarrow L^{q}\left(v, l_{n}\right)} /\|T\|_{L^{p \rightarrow L^{q}}}: T \in B\left(L^{p}(\mu), L^{q}(v)\right), T \neq 0\right\} .
$$

Of course with our notations we have $\gamma=K_{L^{p}(\mu), L^{q}(v)}^{(2)}(2)$.

It is easy to see that if $p=q=r$, then the three above expressions are equal to 1 .

MARCINKIEWICZ and ZYGMUND [28] proved that $K_{p, p}(2)=1$. KRIVINE proved in [20] and [21] that $K_{\infty, 1}^{(2)}(2)=\sqrt{2}$, and in [19] that $K_{p, q}^{(n)}(2) \leq K_{\infty, 1}^{(n)}(2):=K_{G}(n) \leq \lim K_{G}(n):=K_{G}$, where $K_{G}$ is the celebrated Grothendieck constant.

The purpose of this paper is to study systematically the numbers $K_{p, q}^{(n)}(r)$ especially when $r \neq 2$. This paper is divided into seven parts.

Section 1 contains some preliminaries with definitions and some results. These preparations are necessary for later calculations.

In Section 2 our main results are the monotonicity properties of the expressions $K_{p, q}^{(n)}(r)$ and $K_{p, q}(r)$. First we prove that the functions $K_{p, q}^{(n)}(r)$ and $K_{p, q}(r)$ are increasing in $p$ and decreasing in $q$. In particular from this it follows that if $p \leq r \leq q$, then $K_{p, q}(r)=1$. Second we prove the duality formulas $K_{p, q}^{(n)}(r)=K_{q^{\prime}, p^{\prime}}^{(n)}\left(r^{\prime}\right)$ and $K_{p, q}(r)=K_{q^{\prime}, p^{\prime}}\left(r^{\prime}\right)$. It is also proved that $K_{p, q}(r)$ as a function of $r$ is decreasing on [1,2] and increasing on [2, $)$.

In Section 3 we investigate the cases when the constants $K_{p, q}(r)$ are equal to 1 . As note above, MARCINKIEWICZ and ZYGMUND [28] proved that $K_{p, p}(2)=1$, while HeRZ [14] proved 
that $K_{p, p}(r)=1$ for $1<p<\infty$ and $\min (p, 2) \leq r \leq \max (p, 2)$. We give here a little more information on the equality $K_{p, q}(r)=1$. Such equality is mainly connected with the assumption that $p \leq q$. In this section we also give estimates of $K_{p, q}(r)$ in the case when $p>q$; sometimes we obtain even equalities like $K_{2, q}(2)=d_{2}(2) / d_{q}(2)$. For $r \neq 2$ there also possible situations such that $K_{p, q}(r)=\infty$.

In Section 4 we prove estimates for the case $n=2$. Our main result, namely that $K_{\infty, 1}^{(2)}(r)=\max \left(2^{1 / r}, 2^{1-1 / r}\right)$, is a generalization of the KRIVINE result [21] which was proved by him only for $r=2$.

In Section 5 we give estimates of $K_{p, q}^{(n)}(2)$. In particular, the relation between the norms of the real operator and its complexification appears. These types of questions are important in the real-version of the Riesz-Thorin interpolation theorem (see [3], [35], [44], and [46]). In the "lower triangle" the real version of this theorem still holds with the constant 1 and in the "upper triangle" with the constant not bigger than $\sqrt{2}$.

In Section 6 we consider instead of the constants $K_{p . q}^{(n)}(r)$ the more generals ones, namely $K_{X, Y}^{(n)}(r)$, where $L^{p}$ and $L^{q}$ spaces are replaced by arbitrary Banach lattices $X$ and $Y$. It is interesting to note that we always have $K_{X, Y}^{(2)}(r) \leq \max \left(2^{1-1 / r}, 2^{1 / r}\right)$ and that there exists a symmetric two-dimensional Banach space $X$ such that $K_{X}^{(2)}(2)>1$.

Finally, Section 7 is reserved for some additional results and remarks. Specifically we discuss the case when the Banach spaces are complex spaces and present some alternative proofs and possible generalizations. Moreover, we consider and discuss some known estimates of the (famous) Grothendieck constants $K_{G}, K_{G}(n)$ in the real case and $K_{G}^{C}, K_{G}^{C}(n)$ in the complex case.

Some open problems are posed in Sections 2-6.

\section{Preliminaries}

A) For $0<p \leq \infty$ and $a_{1}, a_{2}$ arbitrary real numbers it is easy to prove that

$$
\left(\int_{0}^{1}\left|a_{1} \cos 2 \pi t+a_{2} \sin 2 \pi t\right|^{p} \mathrm{~d} t\right)^{1 / 2}=d_{p}^{(2)}\left(a_{1}^{2}+a_{2}^{2}\right)^{1 / 2},
$$

where $d_{p}^{(2)}=\left(\int_{0}^{1}|\cos 2 \pi t|^{p} \mathrm{~d} t\right)^{1 / p}$.

The equality (1) for $0<p<\infty$ was generalized by STECHKIN [41], in the following lemma:

Lemma 1. Let $0<p<\infty$ and let $n=2,3, \ldots$ be fixed. Then there exists a sequence of orthogonal functions (spherical coordinates) $\left\{\alpha_{k}\right\}_{k=1}^{n}$ such that

$$
\left(\int_{0}^{1}\left|\sum_{k=1}^{n} a_{k} \alpha_{k}(t)\right|^{p} \mathrm{~d} t\right)^{1 / p}=d_{p}^{(n)}\left(\sum_{k=1}^{n} a_{k}^{2}\right)^{1 / 2},
$$

for every sequence of real numbers $\left\{a_{k}\right\}_{k=1}^{n}$, where

$$
d_{p}^{(n)}=[\Gamma((p+1) / 2) \Gamma(n / 2) /(\sqrt{\pi} \Gamma((p+n) / 2))]^{1 / p} .
$$

Observe that $d_{p}^{(n)}$ is increasing in $p, \lim _{p \rightarrow \infty} d_{p}^{(n)}=1$, and $1=d_{p}^{(1)} \geq d_{p}^{(2)} \geq \ldots \geq 0$. Moreover, if $p$ is finite, then $\lim _{n \rightarrow \infty} n^{1 / 2} d_{p}^{(n)}=\sqrt{2}^{p \rightarrow \infty}[\Gamma((p+1) / 2) / \sqrt{\pi}]^{1 / p}$. 
In the case $p=\infty$ it can be shown that $L^{\infty}[0,1]$ contains an isometric copy of $l^{2}$. This means that equality (2) still holds (maybe only with different sequences of functions).

B) We also need to use the $r$-stable Gaussian variables. A random (Gaussian) variable $\omega$ is said to be $r$-stable $(0<r \leq 2)$, if its Fourier transform on $\mathbb{R}$ is equal to exp $\left(-|x|^{r}\right)$. The importance of $r$-stable random variables becomes clear in equation (3) below. It means that the space $l^{r}$ is isometric to a subspace of $L^{p}[0,1]$ for $1 \leq p<r \leq 2$. This result is contained implicitly in LEVY [20] and MARCINKIEWICZ-ZYGMUND [28] (see also KAWATA [16] or PIETSCH [32], p. 289).

Lemma 2. Let either $r=2$ and $0<p<\infty$ or $0<p<r<2$. If $\left\{\omega_{k}\right\}$ is a sequence of identically distributed independent $r$-stable random variables defined on the interval $[0,1]$ with the Lebesgue measure, then

$$
\left(\int_{0}^{1}\left|\sum_{k=1}^{n} a_{k} \omega_{k}(t)\right|^{p} \mathrm{~d} t\right)^{1 / p}=d_{p}(r)\left(\sum_{k=1}^{n}\left|a_{k}\right|^{r}\right)^{1 / r}
$$

for every sequence of real numbers $\left\{a_{k}\right\}_{k=1}^{n}$ and any $n=2,3, \ldots$, where

$$
d_{p}(r)=2\left[\Gamma(1-p / r) \Gamma((p+1) / 2) /(\sqrt{\pi} \Gamma((1-p / 2))]^{1 / p} .\right.
$$

In particular, $d_{p}(2)=2[\Gamma((p+1) / 2) / \sqrt{\pi}]^{1 / p}, d_{2}(2)=\sqrt{2}, d_{1}(2)=2 / \sqrt{\pi}$. Observe also that $d_{p}(r)$ is increasing in $p$ and decreasing in $r$.

The equality (3) for $r=2$ is the Gaussian analogue of the Khinthine inequality for the Rademacher functions.

C) Let $\left\{\boldsymbol{r}_{k}\right\}$ be a sequence of Rademacher functions defined by

$$
r_{k}(t)=\operatorname{sign}\left(\sin 2^{k} \pi t\right), \quad k=0,1, \ldots, 0 \leq t \leq 1 .
$$

Lemma 3. The equality

$$
\left\|\sum_{k=1}^{n} a_{k} r_{k}(t)\right\|_{\infty}=\sum_{k=1}^{n}\left|a_{k}\right|
$$

holds for every sequence of real numbers $\left\{a_{k}\right\}_{k=1}^{n}$ and any $n=1,2,3, \ldots$.

Proof. It is sufficient to note that $\left\|\sum a_{k} r_{k}(t)\right\|_{\infty}=\max _{ \pm}\left|\sum \pm a_{k}\right|$ and $\max _{ \pm}| \pm a \pm b|$ $=|a|+|b|$.

D) Let $X$ be a Banach lattice and $1 \leq p \leq \infty$. For $\left\{x_{k}\right\}_{k=1}^{n} \in X$ we define

$$
\left(\sum_{k=1}^{n}\left|x_{k}\right|^{p}\right)^{1 / p}=\sup \left\{\sum_{k=1}^{n} \lambda_{k} x_{k}: \lambda_{k} \in \mathbb{R}, \sum_{k=1}^{n}\left|\lambda_{k}\right|^{p^{\prime}} \leq 1\right\},
$$

where the supremum means the least upper bound for the lattice structure of $X$. By the Yudin theorem (cf. KRIVINE [19], Th. 1 and Lindenstrauss-TZAFriri [25], Th. 1.d.1) we have that (3) holds for $x_{1}, x_{2}, \ldots, x_{n} \in X$, i.e.,

$$
\left(\int_{0}^{1}\left|\sum_{k=1}^{n} x_{k} \omega_{k}(t)\right|^{p} \mathrm{~d} t\right)^{1 / p}=d_{p}(r)\left(\sum_{k=1}^{n}\left|x_{k}\right|^{r}\right)^{1 / r} \text {. }
$$

The statements (1) and (2) can be generalized in a simililar way. 
E) We shall also need some inequalities for real and complex numbers. First we mention the following generalized version of an inequality of Clarkson type proved by MALIGRANDA and PERSSON [27]:

Lemma 4. If $1 \leq p, q \leq \infty$, then

$$
\left(|a+b|^{q}+|a-b|^{q}\right)^{1 / q} \leq C_{p, q}\left(|a|^{p}+|b|^{p}\right)^{1 / p}
$$

for any complex numbers $a, b$, where $C_{p, q}=\max \left\{2^{1-1 / p}, 2^{1 / q}, 2^{1 / q-1 / p+1 / 2}\right\}$ are the best possible constants.

Next we remark that an inequality for the numbers in $[0,1]$ in the case of $p \geq 2$ was applied by HERz [14]. Here we state and prove that this inequality holds also in the case $1 \leq p \leq 2$.

Lemma 5. Let $1 \leq p<\infty$. Then for any $0 \leq x \leq 1$

$$
(1+x)^{p}+(1-x)^{p} \leq c_{p} x^{\min (p, 2)}+2,
$$

where

$$
c_{p}=2^{p-1} \quad \text { if } p \leq 2 \text {, and } c_{p}=2^{p-2} p(p-1) \text { if } p \geq 2 \text {. }
$$

Proof. Let

$$
f(x)=(1+x)^{p}+(1-x)^{p}-c_{p} x^{\min (p, 2)} .
$$

If $1 \leq p \leq 2$, then

$$
f^{\prime}(x)=p\left[(1+x)^{p-1}-(1-x)^{p-1}-2^{p-1} x^{p-1}\right]
$$

and since

$$
(1+x)^{p-1}=(1-x+2 x)^{p-1} \leq(1-x)^{p-1}+(2 x)^{p-1}
$$

it follows that $f^{\prime}(x) \leq 0$. Thus $f(x) \leq f(0)=2$.

If $p \geq 2$, then

$$
f^{\prime}(x)=p\left[(1+x)^{p-1}-(1-x)^{p-1}-2^{p-1}(p-1) x\right]
$$

and

$$
f^{\prime \prime}(x)=p(p-1)\left[(1+x)^{p-2}+(1-x)^{p-2}-2^{p-1}\right] .
$$

For the function

$$
g(x)=(1+x)^{p-2}+(1-x)^{p-2}
$$

we find that

$$
g^{\prime}(x)=(p-2)\left[(1+x)^{p-3}-(1-x)^{p-3}\right],
$$

and thus

$$
g^{\prime}(x) \leq 0 \quad \text { if } \quad 2 \leq p \leq 3
$$

and

$$
g^{\prime}(x)>0 \text { if } p>3
$$


Therefore

$$
g(x) \leq \max [g(0), g(1)]=\max \left(2,2^{p-2}\right) \leq 2^{p-1},
$$

which means $f^{\prime \prime}(x) \leq 0$, and so $f^{\prime}(x) \leq f^{\prime}(0)=0$. Finally this yields

$$
f(x) \leq f(0)=2
$$

and the proof is complete.

\section{Some properties of $K_{p, q}^{(n)}(r)$ and $K_{p, q}(r)$}

Next we prove the monotonicity of the numbers $K_{p, q}^{\langle n\rangle}(r)$ as a function of $p$ and $q$. It is obvious that these numbers are increasing in $n$.

Theorem 1. (a) The following duality formula hold:

$$
K_{p, q}^{(n)}(r)=K_{p^{\prime}, q^{\prime}}^{(n)}\left(r^{\prime}\right) \quad \text { and } \quad K_{p, q}(r)=K_{q^{\prime}, p^{\prime}}\left(r^{\prime}\right) .
$$

(b) $K_{p, q}^{(n)}(r)$ and $K_{p, q}(r)$ are increasing in $p$ and decreasing in $q$. In particular $K_{p, q}^{(n)}(r) \leq K_{\infty, 1}^{(n)}(r)$.

(c) $K_{p . q}(r)$ as a function of $r$ is decreasing on [1,2] and increasing on $[2, \infty]$.

Before the proof of this theorem let us consider the numbers $K_{p, q}^{(n)}(r)$ as the tensor-product norms of some projections.

Let

$$
\mathbf{S}_{n}^{r}=\left\{\left(x_{1}, \ldots, x_{n}\right) \in \mathbb{R}^{n}: \sum_{k=1}^{n}\left|x_{k}\right|^{r}=1\right\}
$$

be the unit sphere in $l_{n}^{r}$ and let $C_{p}\left(\mathbf{S}_{n}^{r}\right)$ be the linear space of all real-valued continuous functions defined on $\mathbf{S}_{n}^{r}$ with the $L^{\boldsymbol{p}}$-norm, i.e., for any continuous function $f: \mathbf{S}_{n} \rightarrow \mathbb{R}$

$$
\|f\|_{p}=\left(\int_{\mathbf{S}_{n}^{r}}|f(x)|^{p} \mathrm{~d} m_{r}(x)\right]^{1 / p},
$$

where $m_{r}$ is the normalized Lebesgue measure on $\mathbf{S}_{n}^{r}, m_{r}\left(\mathbf{S}_{n}^{r}\right)=1$.

We will calculate the numbers $K_{p, q}^{(n)}(r)$ by using the projections $P_{k}: \mathbf{S}_{n}^{r} \rightarrow[-1,1]$ and $Q_{k}: \mathbf{S}_{n}^{r^{\prime}} \rightarrow[-1,1]$ given by

$$
P_{k}\left(x_{1}, \ldots, x_{n}\right)=x_{k} \quad \text { and } \quad Q_{k}\left(y_{1}, \ldots, y_{n}\right)=y_{k} \quad(k=1,2, \ldots, n) .
$$

The idea of the proof is taken from Krivine [21, Prop. 2].

Let us recall that if $K_{1}, K_{2}$ are compact sets and $C\left(K_{1}\right), C\left(K_{2}\right)$ are normed spaces of continuous functions with the norms \|\|$_{1},\|\|_{2}$, then $C\left(K_{1}\right) \otimes C\left(K_{2}\right)$ denotes the real subspace of $C\left(K_{1} \times K_{2}\right)$ generated by linear combinations of the type $f(x) g(y)$ with $f \in C\left(K_{1}\right)$ and $g \in C\left(K_{2}\right)$.

For any $F \in C\left(K_{1}\right) \otimes C\left(K_{2}\right)$ we define its norm as

$$
\|F\|_{\otimes}=\inf \sum_{k=1}^{m}\left\|f_{k}\right\|_{1}\left\|g_{k}\right\|_{2},
$$


where the infimum is taken over all representations of $F(x, y)$ as

$$
F(x, y)=\sum_{k=1}^{m} f_{k}(x) g_{k}(y)
$$

$f_{k} \in C\left(K_{1}\right), \quad g_{k} \in C\left(K_{2}\right), \quad k=1,2, \ldots, m$ and all $m=1,2, \ldots$ The completion of $C\left(K_{1}\right) \otimes C\left(K_{2}\right)$ with respect to the above norm is the projective tensor product $C\left(K_{1}\right) \hat{\otimes} C\left(K_{2}\right)$ of $C\left(K_{1}\right)$ and $C\left(K_{2}\right)$.

The dual of this space is identified with the bounded operators $T$ from $C\left(K_{1}\right)$ into $C\left(K_{2}\right)^{*}$. For such a given operator $T$, a linear form $U_{T}$ can be defined by

$$
\left\langle U_{T}, f \otimes g\right\rangle=\langle T f, g\rangle \text { for } f \in C\left(K_{1}\right), \quad g \in C\left(K_{2}\right) .
$$

For $A \subset \mathbf{S}_{n}^{r}, B \subset \mathbf{S}_{n}^{r^{\prime}}$ and for $F \in C_{p}\left(\mathbf{S}_{n}^{r}\right) \otimes C_{q}\left(\mathbf{S}_{n}^{r^{\prime}}\right)$ we denote by $\left.F\right|_{A \times B}$ the restriction of $F$ to $A \times B$.

Proposition 1. Let $F \in C_{p}\left(\mathbf{S}_{n}^{r}\right) \hat{\otimes} C_{q}\left(\mathbf{S}_{n}^{\prime}\right)$.

(a) If $1 \leq p \leq \infty$ and $q=\infty$, then

$$
\|F\|_{\otimes}=\sup \left\{\left\|\left.F\right|_{\mathbf{s}_{n}^{r} \times B}\right\|_{\otimes}: B \subset \mathbf{S}_{n}^{\prime}, B \text { finite }\right\} .
$$

(b) If $p=q=\infty$, then

$$
\|F\|_{\otimes}=\sup \left\{\left\|\left.F\right|_{A \times B}\right\|_{\otimes}: A \subset \mathbf{S}_{n}^{r}, B \subset \mathbf{S}_{n}^{r^{\prime}}, \text { A finite, } B \text { finite }\right\} .
$$

(c) If $1 \leq p, q<\infty$, then

$$
\|F\|_{\otimes}=\sup \left\{\left\|\left.F\right|_{A \times B}\right\|_{\otimes}: A \subset \mathbf{S}_{n}^{r}, B \subset \mathbf{S}_{n}^{r^{\prime}}, m_{r}(A)>0, m_{r^{\prime}}(B)>0\right\} .
$$

Proof. (a) Since $\mathbf{S}_{n}^{\prime}$ is a compact subset of $\mathbb{R}^{n}$, it follows that for a given $k=1,2, \ldots$, there is a finite subset. $D_{k}$ of $\mathbf{S}_{n}^{\mathbf{r}^{\prime}}$ such that for any $x \in \mathbf{S}_{n}^{r^{\prime}}$ there exists $y \in D_{k}$ with the distance in $\mathbb{R}^{n}$ not bigger than $2^{-k}$. Define

$$
B_{1}=D_{1} \quad \text { and } \quad B_{k}=D_{1} \cup D_{2} \cup \ldots \cup D_{k} \text { for } k=2,3, \ldots \text {. }
$$

Then each $B_{k}$ is finite and $\lim _{k \rightarrow \infty}\left\|\left.F\right|_{\mathbf{s}_{n}^{r} \times \mathbf{B}_{k}}\right\|_{\otimes}=\|F\|_{\otimes}$.

The proofs of (b) and (c) are similar.

Proposition 2. The following equality holds

$$
K_{p, q}^{(n)}(r)=\left\|\sum_{k=1}^{n} P_{k} \otimes Q_{k}\right\|_{C_{p}\left(\mathbf{S}_{n}^{r}\right) \otimes \mathbf{C}_{q^{\prime}}\left(\mathbf{S}_{n}^{r}\right)} .
$$

Proof. First let us consider the case $p=\infty$ and $q=1$.

By Proposition 1(a), for any $\varepsilon>0$ there is a finite set $B \subset \mathbf{S}_{n}^{r^{\prime}}$ such that

$$
\left\|\sum_{k=1}^{n} P_{k} \otimes Q_{k}\right\|-\varepsilon<\left\|\left.\left(\sum_{k=1}^{n} P_{k} \otimes Q_{k}\right)\right|_{\mathbf{S}_{n}^{r} \times B}\right\| .
$$


By the Hahn-Banach theorem there exists a continous linear functional

$$
H: C_{\infty}\left(\mathbf{S}_{n}^{r}\right) \hat{\otimes} C_{\infty}(B) \rightarrow \mathbb{R} \text { such that }\|H\|=1
$$

and

$$
H\left(\sum_{k=1}^{n} P_{k} \otimes Q_{k}\right)=\left\|\left.\left(\sum_{k=1}^{n} P_{k} \otimes Q_{k}\right)\right|_{\mathbf{S}_{n}^{r} \times B}\right\|
$$

We know that the dual of $C_{\infty}\left(\mathbf{S}_{n}^{r}\right) \hat{\otimes} C_{\infty}(B)$ can be identified with the bounded linear operators from $C_{\infty}\left(\mathbf{S}_{n}^{r}\right)$ into $C_{\infty}(B)^{*}$. Therefore there is $T: C_{\infty}\left(\mathbf{S}_{n}^{r}\right) \rightarrow C_{\infty}(B)^{*}$ such that $\|H\|=\|T\|=1$ and $H\left(\sum_{k=1}^{n} P_{k} \otimes Q_{k}\right)=\sum_{k=1}^{n}\left\langle T P_{k}, Q_{k}\right\rangle$. Moreover, since $C_{\infty}\left(\mathbf{S}_{n}^{r}\right)$ is a $L^{\infty}\left(\mathbf{S}_{n}^{r}, \mu\right)$ space for some measure $\mu$, we find that $T$ is an operator on $L^{\infty}\left(\mathbf{S}_{n}^{r}, \mu\right)$ with the same norm, and since $B$ is a finite set, it follows that $C_{\infty}(B)^{*}=L^{1}(B, m)$ with $m(\{b\})=1$ for each $b \in B$ and

$$
\sum_{k=1}^{n}\left\langle T P_{k}, Q_{k}\right\rangle=\sum_{k=1}^{n} \int_{B} T P_{k}(b) Q_{k}(b) \mathrm{d} m(b)=\int_{B} \sum_{k=1}^{n} T P_{k}(b) Q_{k}(b) \mathrm{d} m(b)
$$

Now using the facts that

$$
\sum_{k=1}^{n}\left|P_{k}(b)\right|^{r}=1, \quad \sum_{k=1}^{n}\left|Q_{k}(b)\right|^{r^{\prime}}=1
$$

for each $b \in B,\|T\|=1$ and the Hölder inequality we obtain

$$
\begin{aligned}
\sum_{k=1}^{n}\left\langle T P_{k}, Q_{k}\right\rangle & \leq \int_{B}\left(\sum_{k=1}^{n}\left|T P_{k}(b)\right|^{r}\right)^{1 / r} \mathrm{~d} m(b)=\left\|\left(\sum_{k=1}^{n}\left|T P_{k}(b)\right|^{r}\right)^{1 / r}\right\|_{L^{1}(B, m)} \\
& \leq K_{\infty, 1}^{(n)}(r)\|T\|\left\|\left(\sum_{k=1}^{n}\left|P_{k}\right|^{r}\right)^{1 / r}\right\|_{L^{\infty}\left(\mathbf{S}_{n}^{r}, \mu\right)}=K_{\infty}^{(n), 1}(r) .
\end{aligned}
$$

Hence we conclude that

$$
\left\|\sum_{k=1}^{n} P_{k} \otimes Q_{k}\right\| \leq K_{\infty, 1}^{(n)}(r) .
$$

For the proof in the opposite direction, let us consider any $T: L^{\infty}(X, \mu) \rightarrow L^{1}(Y, v)$, $\|T\|=1$ and $f_{k} \in L^{\infty}(X, \mu), k=1,2, \ldots, n$.

We can suppose that $X, Y$ are finite subsets and $\left\|\left(\sum_{k=1}^{n}\left|f_{k}\right|^{r}\right)^{1 / r}\right\|_{\infty}=1$.

Let $X^{\prime}=\bigcup_{k=1}^{n} \operatorname{supp}\left(f_{k}\right)$ and $Y^{\prime}=\bigcup_{k=1}^{n} \operatorname{supp}\left(U f_{k}\right)$. Define

$$
\begin{aligned}
& J_{1}: L^{\infty}\left(X^{\prime}\right) \rightarrow L^{\infty}(X) \text { by } J_{1}(g)=g\left(\sum_{k=1}^{n}\left|f_{k}\right|^{r}\right)^{1 / r}, \\
& J_{2}: L^{1}(Y) \rightarrow L^{1}\left(Y^{\prime}\right) \text { by } J_{2}(g)=g 1_{Y^{\prime}}
\end{aligned}
$$

and

$$
S: L^{\infty}\left(X^{\prime}\right) \rightarrow L^{1}\left(Y^{\prime}\right) \quad \text { by } \quad S=J_{2} \circ T \circ J_{1} .
$$


For $g_{k}=f_{k} /\left(\sum_{k=1}^{n}\left|f_{k}\right|^{r}\right)^{1 / r} \in L^{\infty}\left(X^{\prime}\right)$ we have $\sum_{k=1}^{n}\left|g_{k}\right|^{r}=1, S g_{k}=J_{2}\left(T\left(J_{1} g_{k}\right)\right)=J_{2}\left(T\left(f_{k}\right)\right)$ $=1_{Y^{\prime}} \cdot T\left(f_{k}\right)$ and

$$
\begin{aligned}
\left\|\left(\sum_{k=1}^{n}\left|T f_{k}\right|^{r}\right)^{1 / r}\right\|_{1} & =\left\|\left(\sum_{k=1}^{n}\left|S g_{k}\right|^{r}\right)^{1 / r}\right\|_{1}=\int_{Y^{\prime}}\left(\sum_{k=1}^{n}\left|S g_{k}\right|^{r}\right)^{1 / r} \mathrm{~d} v \\
& =\int_{Y^{\prime}} \sum_{k=1}^{n}\left|S g_{k}\right| \sum_{k=1}^{n}\left|S g_{k}\right|^{r-1} /\left(\sum_{k=1}^{n}\left|S g_{k}\right|^{r}\right)^{1-1 / r} \mathrm{~d} v \\
& =\int_{Y^{\prime}} \sum_{k=1}^{n} S g_{k} \cdot h_{k} \mathrm{~d} v
\end{aligned}
$$

where

$$
h_{k}=\left|S g_{k}\right|^{r-1}\left(\sum_{k=1}^{n}\left|S g_{k}\right|^{r}\right)^{1 / r-1} \operatorname{sign}\left(S g_{k}\right) \text { with } \sum_{k=1}^{n}\left|h_{k}\right|^{r^{\prime}}=1 .
$$

Now if we consider

$$
S: L^{\infty}\left(X^{\prime}\right)=C\left(X^{\prime}\right) \rightarrow C\left(Y^{\prime}\right)^{*}=L^{1}\left(Y^{\prime}\right),
$$

then

$$
\int_{\mathbf{Y}^{\prime}} \sum_{k=1}^{n} S g_{k} \cdot h_{k} \mathrm{~d} v=\sum_{k=1}^{n}\left\langle S g_{k}, h_{k}\right\rangle \leq\|S\|\left\|\sum_{k=1}^{n} g_{k} \otimes h_{k}\right\|_{C\left(X^{\prime}\right) \otimes C\left(Y^{\prime}\right)} .
$$

Since

$$
\|S\| \leq\left\|T^{\prime}\right\|=1 \text { and } \sum_{k=1}^{n}\left|g_{k}\right|^{r}=\sum_{k=1}^{n}\left|h_{k}\right|^{r^{\prime}}=1
$$

we have

$$
\left\|\sum_{k=1}^{n} g_{k} \otimes h_{k}\right\|_{C\left(X^{\prime}\right) \otimes C\left(Y^{\prime}\right)} \leq\left\|\sum_{k=1}^{n} P_{k} \otimes Q_{k}\right\|_{C_{\infty}\left(\mathbf{S}_{n}^{n}\right) \otimes C_{\infty}\left(\mathbf{S}_{n}^{\prime \prime}\right)}
$$

and the reverse inequality of (6) holds for $q=1$.

Secondly let $p=\infty$ and $1<q<\infty$. Let us write

$$
a=\left\|\sum_{k=1}^{n} P_{k} \otimes Q_{k}\right\|_{C_{\infty}\left(\mathbf{S}_{n}^{r}\right) \otimes C_{q^{\prime}}\left(\mathbf{S}_{n}^{r_{n}^{\prime \prime}}\right)} .
$$

According to Proposition 1(c), for any $\varepsilon>0$ there are subsets $A \subset \mathbf{S}_{n}^{r}, B \subset \mathbf{S}_{n}^{r^{\prime}}$ with $m_{r}(A)>0, m_{r}(B)>0$ such that

$$
\begin{aligned}
& a-\varepsilon<\left\|\left.\left(\sum_{k=1}^{n} P_{k} \otimes Q_{k}\right)\right|_{\boldsymbol{A} \times \boldsymbol{B}}\right\|_{C_{\infty}\left(\mathbf{S}_{n}^{r}\right) \otimes C_{\boldsymbol{q}^{\prime}}\left(\mathbf{S}_{n}^{\prime \prime}\right)} \\
& =\left\|\left.\left(\sum_{k=1}^{n} P_{k} \otimes Q_{k}\right)\right|_{\boldsymbol{A} \times \boldsymbol{B}}\right\|_{\boldsymbol{C}_{\infty}\left(\mathbf{S}_{n}^{r}\right) \otimes L^{\sigma^{\prime}}\left(\mathbf{S}_{n}^{\prime \prime}\right)},
\end{aligned}
$$

where the last equality holds because $C_{q^{\prime}}\left(\mathbf{S}_{n}^{r^{\prime}}\right)$ is dense in $L^{q^{\prime}}\left(\mathbf{S}_{n}^{r^{\prime}}\right)$. There exists also a linear bounded functional

$$
H: C_{\infty}\left(\mathbf{S}_{n}^{r}\right) \hat{\otimes} L^{q^{\prime}}\left(\mathbf{S}_{n}^{r^{\prime}}\right) \rightarrow \mathbb{R}
$$


and an operator

and

$$
T: C_{\infty}\left(\mathbf{S}_{n}^{r}\right) \rightarrow L^{q^{\prime}}\left(\mathbf{S}_{n}^{r^{\prime}}\right)^{*}=L^{q}\left(\mathbf{S}_{n}^{r}\right) \text { such that }\|H\|=\|T\|=1
$$

$$
\begin{aligned}
& \left\|\left.\left(\sum_{k=1}^{n} P_{k} \otimes Q_{k}\right)\right|_{A \times B}\right\|_{C_{\infty}\left(\mathbf{S}_{n^{r}}^{r}\right) \otimes L^{q^{\prime}}\left(\mathbf{S}_{\left.r^{\prime}\right)}^{r^{\prime}}\right.}=H\left(\left.\left(\sum_{k=1}^{n} P_{k} \otimes Q_{k}\right)\right|_{A \times B}\right) \\
& =\left.\sum_{k=1}^{n}\left\langle T P_{k}, Q_{k}\right\rangle\right|_{A \times B}=\int_{B} \sum_{k=1}^{n} T P_{k}(t) Q_{k}(t) \mathrm{d} m_{r^{\prime}} \\
& \leq \int_{B}\left(\sum_{k=1}^{n}\left|T P_{k}(t)\right|^{r}\right)^{1 / r}\left(\sum_{k=1}^{n}\left|Q_{k}(t)\right|^{r^{\prime}}\right)^{1 / r^{\prime}} \mathrm{d} m_{r^{\prime}} \\
& \leq\left(\int_{B}\left(\left.\sum_{k=1}^{n} T P_{k}(t)\right|^{r}\right)^{q / r} \mathrm{~d} m_{r^{\prime}}\right)^{1 / q}\left(\int\left(\sum_{B=1}^{n}\left|Q_{k}(t)\right|^{r^{\prime}}\right)^{q^{\prime} / r^{\prime}} \mathrm{d} m_{r^{\prime}}\right)^{1 / q^{\prime}} \\
& \leq\left\|\left(\sum_{k=1}^{n}\left|T P_{k}(b)\right|^{r}\right)^{1 / r}\right\|\left\|_{L^{q}\left(B, m_{r^{\prime}}\right)} \leq K_{\infty, q}^{(n)}(r)\right\| T\|\|\left(\sum_{k=1}^{n}\left|P_{k}\right|^{r}\right)^{1 / r} \|_{\infty} \leq K_{\infty, q}^{(n)}(r) .
\end{aligned}
$$

Because $\varepsilon>0$ was chosen arbitrarily we conclude that $a \leq K_{\infty, q}^{(n)}(r)$.

Conversely, for any linear bounded operator $T: L^{\infty}(X, \mu) \rightarrow L^{q}(Y, v)$ we shall prove that for all $f_{1}, f_{2}, \ldots, f_{n} \in L^{\infty}(X, \mu)$ the following inequality holds:

$$
\left\|\left(\sum_{k=1}^{n}\left|T f_{k}\right|^{r}\right)^{1 / r}\right\|_{q} \leq a\|T\|\left\|\left(\sum_{k=1}^{n}\left|f_{k}\right|^{r}\right)^{1 / r}\right\|_{\infty} .
$$

We can assume that

$$
\|T\|=.1=\left\|\left(\sum_{k=1}^{n}\left|f_{k}\right|^{r}\right)^{1 / r}\right\|_{\infty}
$$

and that $X$ and $Y$ are finite subsets. Using, as before, the functions $f_{k}, g_{k}(k=1,2, \ldots, n)$ and the operators $J_{1}, J_{2}$ and $S$ with $L^{q}\left(Y^{\prime}\right)$ instead of $L^{1}\left(Y^{\prime}\right)$ we have

$$
\begin{aligned}
\left\|\left(\sum_{k=1}^{n}\left|T f_{k}\right|^{r}\right)^{1 / r}\right\|_{q} & =\left\|\left(\sum_{k=1}^{n}\left|S g_{k}\right|^{r}\right)^{1 / r}\right\|_{q}=\left(\int_{y^{\prime}}\left(\sum_{k=1}^{n}\left|S g_{k}\right|^{r}\right)^{q / r} \mathrm{~d} v\right)^{1 / q} \\
& =\left(\int_{Y^{\prime}}\left(\sum_{k=1}^{n} S g_{k} \cdot h_{k}\right)^{q} \mathrm{~d} v\right)^{1 / q} \\
& =\left(\sum_{y \in Y^{\prime}}\left(\sum_{k=1}^{n} S g_{k}(y) \cdot h_{k}(y)\right)^{q}\right)^{1 / q} \leq\left(\sum_{y \in Y^{\prime}} \sum_{k=1}^{n} S g_{k}(y) \cdot h_{k}(y)\right) \\
& =\int_{Y^{\prime}}\left(\sum_{k=1}^{n} S g_{k} \cdot h_{k}\right) \mathrm{d} v=\sum_{k=1}^{n}\left\langle S g_{k}, h_{k}\right\rangle \\
& \leq\|S\| \sum_{k=1}^{n} g_{k} \otimes h_{k} \|_{L^{\infty}\left(X^{\prime}\right) \otimes L^{q^{\prime}}\left(Y^{\prime}\right)} \\
& \leq\left\|\sum_{k=1}^{n} g_{k} \otimes h_{k}\right\|_{L^{\infty}\left(X^{\prime}\right) \otimes L^{q^{\prime}}\left(Y^{\prime}\right)} \leq a,
\end{aligned}
$$

which implies the reverse inequality $K_{\infty, q}^{(n)}(r) \leq a$. 
Thirdly let $1 \leq p<\infty$ and $1<q<\infty$. Let us write

$$
a=\left\|\sum_{k=1}^{n} P_{k} \otimes Q_{k}\right\|_{C_{p}\left(\mathbf{S}_{n}^{r}\right) \otimes C_{q^{\prime}}\left(\mathbf{S}_{n}^{r^{\prime}}\right)}=\left\|\sum_{k=1}^{n} P_{k} \otimes Q_{k}\right\|_{L^{p}\left(\mathbf{S}_{n}^{r}\right) \otimes L^{q^{\prime}}\left(\mathbf{S}_{n}^{r^{\prime}}\right)} .
$$

The last equality holds true because $C_{p}\left(\mathbf{S}_{n}^{r}\right)$ and $C_{q^{\prime}}\left(\mathbf{S}_{n}^{r^{\prime}}\right)$ are dense in $L^{p}\left(\mathbf{S}_{n}^{r}\right)$ and $L^{q^{\prime}}\left(\mathbf{S}_{n}^{r^{\prime}}\right)$, respectively.

By Proposition 1 (c), for any $\varepsilon>0$ there are $A \subset \mathbf{S}_{n}^{r}, B \subset \mathbf{S}_{n}^{r^{\prime}}$ with $m_{r}(A)>0, m_{r^{\prime}}(B)>0$ such that

$$
a-\varepsilon<\left\|\left.\left(\sum_{k=1}^{n} P_{k} \otimes Q_{k}\right)\right|_{A \times B}\right\|_{L^{p}(A) \otimes L^{q^{\prime}(B)}} .
$$

Then, similarly as in (a), there exists a continuous linear functional

$$
H: L^{p}\left(A, m_{r}\right) \otimes L^{q^{\prime}}\left(B, m_{r^{\prime}}\right) \rightarrow \mathbb{R}
$$

and a continuous linear operator

$$
T: L^{p}\left(A, m_{r}\right) \rightarrow L^{q^{\prime}}\left(B, m_{r^{\prime}}\right)^{*}=L^{q}\left(B, m_{r^{\prime}}\right)
$$

such that $\|H\|=\|T\|=1$ and

$$
\left\|\left.\left(\sum_{k=1}^{n} P_{k} \otimes Q_{k}\right)\right|_{A \times B}\right\|=H\left(\sum_{k=1}^{n} P_{k} \otimes Q_{k}\right)=\sum_{k=1}^{n}\left\langle T P_{k}, Q_{k}\right\rangle .
$$

Since

$$
\begin{aligned}
\sum_{k=1}^{n}\left\langle T P_{k}, Q_{k}\right\rangle & =\int_{B} \sum_{k=1}^{n} T P_{k}(b) Q_{k}(b) \mathrm{d} m_{r^{\prime}} \\
& \leq \int_{B}\left(\sum_{k=1}^{n}\left|T P_{k}(b)\right|^{r}\right)^{1 / r}\left(\sum_{k=1}^{n}\left|Q_{k}(b)\right|^{r^{\prime}}\right)^{1 / r^{\prime}} \mathrm{d} m_{r^{\prime}} \\
& \leq \int_{B}\left(\sum_{k=1}^{n}\left|T P_{k}(b)\right|^{r}\right)^{1 / r} \mathrm{~d} m_{r^{\prime}} \\
& \leq\left(\int_{B}\left(\sum_{k=1}^{n}\left|T P_{k}(b)\right|^{r}\right)^{q / r} \mathrm{~d} m_{r^{\prime}}\right)^{1 / q} m_{r^{\prime}}(B)^{1 / q^{\prime}} \\
& \leq\left\|\left(\sum_{k=1}^{n}\left|T P_{k}(b)\right|^{r}\right)^{1 / r}\right\|_{L^{q}\left(B, m_{\left.r^{\prime}\right)}\right)}^{1 / r} \|_{L^{p^{\prime}(A)}} \leq K_{p, q}^{n}(r),
\end{aligned}
$$

it follows that $a \leq K_{p, q}^{(n)}(r)$. The proof of the reverse inequality is similar as in the second case.

The proofs in the remaining cases $1 \leq p \leq \infty, q=\infty$ and $1 \leq p<\infty, q=1$ are similar and thus omitted.

Proof of the Theorem 1. (a) The duality formula for $K_{p, q}^{(n)}(r)$ follows immediately from (7). Moreover, since the sequence $\left\{K_{p, q}^{(n)}(r)\right\}$ is increasing in $n$,

$$
K_{p, q}(r)=\lim _{n \rightarrow \infty} K_{p, q}^{(n)}(r)=\lim _{n \rightarrow \infty} K_{q^{\prime}, p^{\prime}}^{(n)}\left(r^{\prime}\right)=K_{q^{\prime}, p^{\prime}}\left(r^{\prime}\right) .
$$


(b) Equality (7) also means that

$$
K_{p, q}^{(n)}(r)=\inf \left\{\sum_{i=1}^{m}\left\|f_{i}\right\|_{C_{p}\left(\mathbf{S}_{n}^{r}\right)}\left\|g_{i}\right\|_{C_{q^{\prime}}\left(\mathbf{S}_{n}^{r}\right)}: \sum_{k=1}^{n} P_{k} \otimes Q_{k}=\sum_{i=1}^{m} f_{i} g_{i}, m=1,2, \ldots\right\}
$$

and in this formula we have $L^{p}$ - and $L^{q^{\prime}}$-norms on probability measures spaces. Therefore, $K_{p, q}^{(n)}(r)$ increases with $p$ and decreases with $q$. Then, similarly as in (a), we have also that $K_{p, q}(r)$ increases with $p$ and decreases with $q$.

(c) First let us prove that $C_{r} \leq K_{p, q}(r)$, where $C_{r}$ is the smallest constant $C \geq 1$ such that

$$
\left\|\left(\int_{0}^{1}\left|\sum_{k=1}^{n} T f_{k} g_{k}(t)\right|^{r} \mathrm{~d} t\right)^{1 / r}\right\|_{q} \leq C\|T\|\left\|\left(\int_{0}^{1}\left|\sum_{k=1}^{n} f_{k} g_{k}(t)\right|^{r} \mathrm{~d} t\right)^{1 / r}\right\|_{p}
$$

for all $f_{1}, f_{2}, \ldots, f_{n} \in L^{p}(X, \mu)$ and some $g_{1}, g_{2}, \ldots, g_{n} \in L^{r}(0,1)$, with $n=1,2, \ldots$ arbitrary. We will use in the proof the KRIVINE's idea (cf. [19], Lemma 1). For any $\varepsilon>0$ there are simple functions $s_{k}$ such that $\left\|g_{k}-s_{k}\right\|_{r}<\varepsilon, k=1,2, \ldots, n$. Then

$$
\begin{aligned}
\left|\left\|\sum_{k=1}^{n} f_{k} g_{k}(\cdot)\right\|_{r}-\left\|\sum_{k=1}^{n} f_{k} s_{k}(\cdot)\right\|_{r}\right| & \leq\left\|\sum_{k=1}^{n} f_{k}\left[g_{k}(\cdot)-s_{k}(\cdot)\right]\right\|_{r} \\
& \leq \sum_{k=1}^{n}\left|f_{k}\right|\left\|g_{k}-s_{k}\right\|_{r}<\varepsilon \sum_{k=1}^{n}\left|f_{k}\right|
\end{aligned}
$$

and so

$$
\left\|\sum_{k=1}^{n} f_{k} g_{k}(\cdot)\right\|_{r} \leq\left\|\sum_{k=1}^{n} f_{k} s_{k}(\cdot)\right\|_{r}+\varepsilon \sum_{k=1}^{n}\left|f_{k}\right| \leq\left\|\sum_{k=1}^{n} f_{k} g_{k}(\cdot)\right\|_{r}+2 \varepsilon \sum_{k=1}^{n}\left|f_{k}\right|
$$

The monotonicity of the norm $\|\cdot\|_{p}$ gives

$$
\begin{aligned}
\|\| \sum_{k=1}^{n} f_{k} g_{k}(\cdot)\left\|_{r}\right\|_{p} & \leq\|\| \sum_{k=1}^{n} f_{k} s_{k}(\cdot)\left\|_{r}\right\|_{p}+\varepsilon\left\|\sum_{k=1}^{n}\left|f_{k}\right|\right\|_{p} \\
& \leq\|\| \sum_{k=1}^{n} f_{k} g_{k}(\cdot)\left\|_{r}\right\|_{p}+2 \varepsilon\left\|\sum_{k=1}^{n}\left|f_{k}\right|\right\|_{p} .
\end{aligned}
$$

In the same way, by putting $T f_{k}$ instead of $f_{k}$, we obtain

$$
\begin{aligned}
\|\| \sum_{k=1}^{n} T f_{k} s_{k}(\cdot)\left\|_{r}\right\|_{q} & \leq\|\| \sum_{k=1}^{n} T f_{k} s_{k}(\cdot)\left\|_{r}\right\|_{q}+\varepsilon\left\|\sum_{k=1}^{n}\left|T f_{k}\right|\right\|_{q} \\
& \leq\|\| \sum_{k=1}^{n} T f_{k} g_{k}(\cdot)\left\|_{r}\right\|_{q}+2 \varepsilon\left\|\sum_{k=1}^{n}\left|T f_{k}\right|\right\|_{q} .
\end{aligned}
$$

But

$$
s_{k}=\sum_{i=1}^{m} c_{i, k} 1_{A_{i}}(t)
$$


where $c_{i, k} \in \mathbb{R}$ and $A_{i}$ are measureable subsets of $[0,1](i=1,2, \ldots, m ; k=1,2, \ldots, n)$, therefore

$$
\begin{aligned}
\|\| \sum_{k=1}^{n} T f_{k} g_{k}(\cdot)\left\|_{r}\right\|_{q} & =\left\|\left(\sum_{i=1}^{m}\left|\sum_{k=1}^{n} T f_{k} c_{i, k} m\left(A_{i}\right)^{1 / r}\right|^{r}\right)^{1 / r}\right\|_{q} \\
& =\left\|\left(\sum_{i=1}^{m}\left|T\left(\sum_{k=1}^{n} f_{k} c_{i, k} m\left(A_{i}\right)^{1 / r}\right)\right|^{r}\right)^{1 / r}\right\|_{q} \\
& \leq K_{p, q}(r)\|T\|\left\|\left(\sum_{i=1}^{m}\left|\sum_{k=1}^{n} f_{k} c_{i, k} m\left(A_{i}\right)^{1 / r}\right|^{r}\right)^{1 / r}\right\|_{q} \\
& =K_{p, q}(r)\|T\|\left\|\sum_{k=1}^{n} f_{k} s_{k}(\cdot)\right\|_{r} \|_{p}
\end{aligned}
$$

Hence

$$
\begin{aligned}
\|\| \sum_{k=1}^{n} T f_{k} g_{k}(\cdot)\left\|_{r}\right\|_{q} & \leq\|\| \sum_{k=1}^{n} T f_{k} s_{k}(\cdot)\left\|_{r}+\varepsilon\right\| \sum_{k=1}^{n}\left|T f_{k}\right| \|_{q} \\
& \leq K_{p, q}(r)\|T\|\left\|\sum_{k=1}^{n} f_{k} s_{k}(\cdot)\right\|_{r}\left\|_{p}+\varepsilon\right\| \sum_{k=1}^{n}\left|T f_{k}\right| \|_{q} \\
& \leq K_{p, q}(r)\|T\|\left(\|\| \sum_{k=1}^{n} f_{k} s_{k}(\cdot)\left\|_{r}\right\|_{p}+\varepsilon\left\|\sum_{k=1}^{n}\left|f_{k}\right|\right\|_{p}\right) \\
& +\varepsilon\left\|\sum_{k=1}^{n}\left|T f_{k}\right|\right\| \|_{q} \\
& \leq K_{p, q}(r)\|T\|\left(\left\|\sum_{k=1}^{n} f_{k} s_{k}(\cdot)\right\|_{r}\left\|_{p}+\varepsilon \sum_{k=1}^{n}\right\| f_{k} \|_{p}\right) \\
& +\varepsilon\|T\|\left(\sum_{k=1}^{n}\left\|f_{k}\right\|_{p}\right),
\end{aligned}
$$

and since $\varepsilon>0$ was chosen arbitrarily, we get $C_{r} \leq K_{p, q}(r)$.

Now if $1 \leq r<s \leq 2$, then according to Lemma 2 with $\left(g_{k}\right)=\left(\omega_{k}\right) s$-stable random variables we have

$$
\begin{aligned}
d_{r}(s)\left\|\left(\sum_{k=1}^{n}\left|T f_{k}\right|^{s}\right)^{1 / s}\right\|_{q} & =\left\|\left(\int_{0}^{1}\left|\sum_{k=1}^{n} T f_{k} \omega_{k}(t)\right| \mathrm{d} t\right)^{1 / r}\right\|_{q} \\
& \leq C_{r}\|T\|\left\|\left(\int_{0}^{1}\left|\sum_{k=1}^{n} f_{k} \omega_{k}(t)\right|^{r} \mathrm{~d} t\right)^{1 / r}\right\|_{p} \\
& =C_{r} d_{r}(s)\|T\|\left\|\left(\sum_{k=1}^{n}\left|f_{k}\right|^{s}\right)^{1 / s}\right\|_{p}
\end{aligned}
$$

Thus

$$
\left\|\left(\sum_{k=1}^{n}\left|T f_{k}\right|^{s}\right)^{1 ' s}\right\|_{q} \leq C_{r}\|T\|\left\|\left(\sum_{k=1}^{n}\left|f_{k}\right|^{s}\right)^{1 / s}\right\|_{p},
$$


which together with (8) implies that

$$
K_{p, q}(s) \leq C_{r} \leq K_{p, q}(r) .
$$

The second case (i.e., when $2 \leq r<s \leq \infty$ ) follows from the duality formula.

Problem 1. Let $n \geq 2$ be fixed. Does $K_{p, q}^{(n)}(r) \geq K_{p, q}^{(n)}(2)$ hold, or more generally, is $K_{p, q}^{(n)}(r)$ a decreasing function of $r$ on $[1,2]$ and an increasing function of $r$ on $[2, \infty]$ ?

\section{On the estimates of Marcinkiewicz-Zygmund and Herz type}

MARCINKIEWICZ and ZYGMUND [28] showed that if $1 \leq p<\infty$ then $K_{p, p}(2)=1$, and if $1 \leq p<r<2$ then $K_{p, p}(r)=1$. HeRz [14] proved that for $1<p<\infty, K_{p, p}(r)=1$ if, and only if, $\min (p, 2) \leq r \leq \max (p, 2)$.

The purpose of this section is to utilize ideas of MARCINKIEWICZ-ZYGMUND [28] and HERZ [14] to generalize and improve these results. In particular, these results permit us to analyze $K_{p, q}(r)=1$ also for $p \neq q$.

Theorem 2. (a) If either $p=1$ or $q=\infty$, then $K_{p, q}(r)=1$.

(b) If $1<p \leq q<\infty$ and $\min (p, 2) \leq r \leq \max (q, 2)$, then $K_{p, q}(r)=1$.

(c) If $1<p \leq q<\infty$ and $K_{p, q}(r)=1$, then $\min \left(p, q^{\prime}\right) \leq r \leq \max (p, q)$.

(d) If $1<p<\infty$ and $K_{p, p}(r)=1$, then $\min (p, 2) \leq r \leq \max (p, 2)$.

(e) $K_{p, q}(2)=1$ if, and only if, $p \leq q$.

Remark 1. If $p \leq q$, then $[\min (p, 2), \max (p, 2)] \subset\left[\min \left(p, q^{\prime}\right), \max \left(p^{\prime}, q\right)\right]$.

Before we prove Theorem 2, we investigate when the appropriate constants are 1 in the case $n=2$.

Proposition 3. (a) The equality $K_{l_{2}^{2}, l_{2}^{(}}^{(2)}(2)=1$ holds if, and only if, either $p \leq 2$ or $q \geq 2$.

(b) Let either $p \leq 2$ or $q \geq 2$. If $K_{p, q}^{(2)}(r)=1$, then $\min \left(p, q^{\prime}\right) \leq r \leq \max \left(p^{\prime}, q\right)$.

(c) If $1<p<\infty$ and $K_{p, p}^{(2)}(r)=1$, then $\min (p, 2) \leq r \leq \max (2, p)$.

Proof. (a $\Leftarrow$ ) (cf. [47], [11]). Let either $p \leq 2$ or $q \geq 2$. An arbitrary operator $T: l_{2}^{p} \rightarrow l_{2}^{q}$ is given by the matrix $\left(\begin{array}{ll}a & b \\ c & d\end{array}\right)$. If $a b c d \geq 0$, then the norm of $T_{c}$ is attained on certain real vector which means that $\left\|T_{c}\right\|_{p, q}=\|T\|_{p, q}$.

Let $a b c d<0$ and let $q \geq 2$. Assume that the norm of the operator $T_{c}$ is attained on the vector $z=(x, y)$ with $y \neq 0$. Put $y / x=\varrho(\cos \varphi+i \sin \varphi)$ and consider a function

$$
\Phi_{\ell}(\varphi)=\left(a^{2}+2 a b \varrho \cos \varphi+b^{2} \varrho^{2}\right)^{q / 2}+\left(c^{2}+2 c d \varrho \cos \varphi+d^{2} \varrho^{2}\right)^{q / 2} .
$$

In the interval $(0, \pi)$ there is at most one extremal point of $\Phi_{Q}(\varphi)$ in which the derivative

$$
\begin{aligned}
\Phi_{\varrho}^{\prime}(\varphi)=-q \varrho \sin \varphi & {\left[a b\left(a^{2}+2 a b \varrho \cos \varphi+b^{2} \varrho^{2}\right)^{q / 2-1}\right.} \\
& \left.+c d\left(c^{2}+2 c d \varrho \cos \varphi+d^{2} \varrho^{2}\right)^{q / 2-1}\right]
\end{aligned}
$$

changes its sign from (-) to (+). Therefore the maximum of $\Phi_{\varrho}(\varphi)$ is at $\varphi=0$ or $\varphi=\pi$, i.e., the vector $z$ is real. 
Therefore we have proved that if $q \geq 2$, then $\left\|T_{C}\right\|_{p, q}=\|T\|_{p, q}$. Then case when $p \leq 2$ can be proved by using the dual operators.

$(\mathrm{a} \Rightarrow$ ) To have such necessary conditions on indices $p$ and $q$ we should consider concrete operators.

Example 1. Let an operator $T: l_{2}^{p} \rightarrow l_{2}^{q}$ be given by the matrix $\left(\begin{array}{rr}1 & 1 \\ 1 & -1\end{array}\right)$. From Lemma 4 we have that the norm of the above operator between complex spaces is

$$
\left\|T_{C}\right\|_{p, q}=\max \left\{2^{1-1 / p}, 2^{1 / p}, 2^{1 / q-1 / p+1 / 2}\right\} .
$$

Here we also see that if either $p \leq 2$ or $q \geq 2$, then this is also the norm in the real case $\|T\|_{p, q}=\max \left\{2^{1-1 / p}, 2^{1 / q}\right\}$. On the other hand, if $q<2<p$, then some calculations show that

$$
\|T\|_{p, q}=\sup _{0 \leq x \leq 1} \frac{\left[(x+1)^{q}+(1-x)^{q}\right]^{1 / q}}{\left(x^{p}+1\right)^{1 / p}}<2^{1 / q-1 / p+1 / 2}=\left\|T_{C}\right\|_{p, q} .
$$

Therefore if $K_{l_{2}^{2}, l_{2}^{q}}^{(2)}(2)=1$, then either $p \leq 2$ or $q \geq 2$.

(b) Let either $p \leq 2$ or $q \geq 2$. Then for the operator $T$ given in Example 1 we have

$$
\|T\|_{p, q}=\max \left\{2^{1-1 / p}, 2^{1 / q}\right\} \text {. }
$$

For $f_{1}=\left(a_{1}, b_{1}\right)$ and $f_{2}=\left(a_{2}, b_{2}\right)$ we have

$$
\begin{aligned}
& \left\|\left(\left|T f_{1}\right|^{\boldsymbol{r}}+\left|T f_{2}\right|^{\boldsymbol{r}}\right)^{1 / \boldsymbol{r}}\right\|_{q} \\
& =\left[\left(\left|a_{1}+b_{1}\right|^{r}+\left|a_{2}+b_{2}\right|^{r}\right)^{q / r}+\left(\left|a_{1}-b_{1}\right|^{r}+\left|a_{2}-b_{2}\right|^{r}\right)^{q / r}\right]^{1 / q}
\end{aligned}
$$

and

$$
\left\|\left(\left|f_{1}\right|^{r}+\left|f_{2}\right|^{r}\right)^{1 / r}\right\|_{p}=\left[\left(\left|a_{1}\right|^{r}+\left|a_{2}\right|^{r}\right)^{p / r}+\left(\left|b_{1}\right|^{r}+\left|b_{2}\right|^{r}\right)^{p / r}\right]^{1 / p}
$$

Now by considering concrete $f_{1}$ and $f_{2}$ we obtain the desired results. For example if $f_{1}=(0,1)$ and $f_{2}=(1,0)$, then

$$
K_{p, q}^{(2)}(r) \geq\left(2^{q / r}+2^{q / r}\right)^{1 / q} /\left(2^{1 / p}\|T\|_{p, q}\right)=\min \left\{2^{1 / q+1 / r-1}, 2^{1 / r-1 / p}\right\} ;
$$

and if $f_{1}=(1,1)$ and $f_{2}=(1,-1)$, then

$$
K_{p, q}^{(2)}(r) \geq\left(2^{q}+2^{q}\right)^{1 / q} /\left[\left(2^{p / r}+2^{p / r}\right)^{1 / p}\|T\|_{p, q}\right]=\min \left\{2^{1 / q-1 / r}, 2^{-1 / p-1 / r}\right\} .
$$

Therefore, if $K_{p, q}^{(2)}(r)=1$, then $\min \left(p, q^{\prime}\right) \leq r \leq \max \left(p^{\prime}, q\right)$.

(c) We want to prove that if $K_{p, p}^{(2)}(r)=1$, then $r \in[\min (p, 2), \max (p, 2)]$. Again we require some specific operator. The idea in the construction below is taken from HERz $[14$, Lemma 2].

Example 2. If $r<\min (p, 2)$ and $p<\infty$, then there exists an operator $T: l_{2}^{p} \rightarrow l_{3}^{p}$ such that for $T_{E}: l_{2}^{p}\left[l_{2}^{r}\right] \rightarrow l_{3}^{p}\left[l_{2}^{r}\right]$ we have $\|T\|<\left\|T_{E}\right\|$, i.e., $K_{p, p}^{(2)}(r)>1$.

Proof. For $\alpha, \beta>0$ let us consider the operators $T=T_{\alpha, \beta}: l_{2}^{p} \rightarrow l_{3}^{p}$ given by the matrix

$$
\left(\begin{array}{rr}
1 & \alpha \\
1 & -\alpha \\
0 & \beta
\end{array}\right)
$$


Then

$$
\begin{aligned}
\left\|T_{\alpha, \beta}\right\|_{p, p}= & \sup \left\{\left(|x+\alpha y|^{p}+|x-\alpha y|^{p}+|\beta y|^{p}\right)^{1 / p}: x, y \in \mathbb{R}\right. \text { and } \\
& \left.|x|^{p}+|y|^{p}=1\right\} .
\end{aligned}
$$

For a given $\alpha, 0<\alpha<1$, there exists $\beta$ with $0<\beta<2$ such that the supremum is attained at $x=y=2^{-1 / p}$. Therefore

$$
\left\|T_{\alpha, \beta}\right\|_{p, p}=2^{-1 / p}\left[(1+\alpha)^{p}+(1-\alpha)^{p}+\beta^{p}\right]^{1 / p} .
$$

For $f=(\alpha, 0)$ and $g=(0, \alpha)$ we have

and

$$
\left\|\left(|f|^{r}+|g|^{r}\right)^{1 / r}\right\|_{p}=\|(\alpha, \alpha)\|_{p}=\alpha 2^{1 / p},
$$

Thus

$$
\begin{aligned}
\left\|\left(\left|T_{\alpha, \beta} f\right|^{r}+\left|T_{\alpha, \beta} g\right|^{r}\right)^{1 / r}\right\|_{p} & =\left\|\left(\left(\alpha^{r}+\alpha^{2 r}\right)^{1 / r},\left(\alpha^{r}+\alpha^{2 r}\right)^{1 / r}, \alpha \beta\right)\right\|_{p} \\
& =\left[2\left(\alpha^{r}+\alpha^{2 r}\right)^{p / r}+\alpha^{p} \beta^{p}\right]^{1 / p} .
\end{aligned}
$$

$$
\begin{aligned}
& \left\|\left(T_{\alpha, \beta}\right)_{E}\right\|_{p, p} /\left\|T_{\alpha, \beta}\right\|_{p, p} \\
& \geq\left[2\left(\alpha^{r}+\alpha^{2 r}\right)^{p / r}+\alpha^{p} \beta^{p}\right]^{1 / p} /\left\{\alpha\left[(1+\alpha)^{p}+(1-\alpha)^{p}+\beta^{p}\right]^{1 / p}\right\} \\
& =\left\{\left[2\left(1+\alpha^{r}\right)^{p / r}+\beta^{p}\right] /\left[(1+\alpha)^{p}+(1-\alpha)^{p}+\beta^{p}\right]\right\}^{1 / p} .
\end{aligned}
$$

Assume that $r<\min (p, 2)$ and $\left\|\left(T_{\alpha, \beta}\right)_{E}\right\|_{p, p}=\left\|T_{\alpha, \beta}\right\|_{p, p}$. Then

$$
2\left(1+\alpha^{r}\right)^{p / r}+\beta^{p} \leq(1+\alpha)^{p}+(1-\alpha)^{p}+\beta^{p}
$$

or

$$
2\left(1+\alpha^{r}\right)^{p / r} \leq(1+\alpha)^{p}+(1-\alpha)^{p} .
$$

Applying Lemma 5, we obtain

$$
2\left(1+\alpha^{r}\right)^{p / r} \leq c_{p} \alpha^{\min (p, 2)}+2,
$$

which cannot hold for small $\alpha$ since

$$
\lim _{\alpha \rightarrow 0^{+}}\left[2\left(1+\alpha^{r}\right)^{p / r}-2\right] / \alpha^{\min (p, 2)}=p \lim _{\alpha \rightarrow 0^{+}}\left[2\left(1+\alpha^{r}\right)^{p / r-1}\right] / \alpha^{\min (p, 2)-r}=\infty .
$$

It was proved here that if $K_{p, p}^{(2)}(r)=1$, then $\min (p, 2) \leq r$. The duality formula and the proved result show also that if $K_{p, p}^{(2)}(r)=1$, then $r \leq \max (p, 2)$. Thus if $K_{p, p}^{(2)}(r)=1$, then $\min (p, 2) \leq r \leq \max (p, 2)$.

Proof of Theorem 2. (a) Let $q=\infty$. Then according to Lemma 3 we obtain $K_{\infty, \infty}(1)=1$. In fact,

$$
\begin{aligned}
\left\|\sum_{k=1}^{n}\left|T f_{k}\right|\right\|_{\infty} & =\|\| \sum_{k=1}^{n} T f_{k} r_{k}(t)\left\|_{\infty}\right\|_{\infty} \\
& =\left\|\vec{T}\left(\sum_{k=1}^{n} f_{k} r_{k}(t)\right)\right\|_{\infty} \|_{\infty} \\
& \leq\|T\|\|\| \sum_{k=1}^{n} f_{k} r_{k}(t)\|\|_{\infty} \|_{\infty} \\
& =\|T\|\left\|\sum_{k=1}^{n}\left|f_{k}\right|\right\|_{\infty} .
\end{aligned}
$$


Since $K_{\infty, \infty}(\infty)=1$ and $K_{\infty, \infty}(1)=1$, it follows from Theorem 1 (c) that $K_{\infty, \infty}(r)=1$ for all $r, 1 \leq r \leq \infty$. Hence using Theorem 1 (b) we obtain that $K_{p, \infty}(r)=1$ for $1 \leq p, r \leq \infty$. The equality $K_{1, q}(r)=1$ can be obtained from an immediate application of Theorem 1(a) to $2^{0}$.

(b) First observe that if $p \leq r \leq q$, then $K_{p, q}(r)=1$. In fact, using Theorem 1(b) we find that

$$
K_{p, q}(r) \leq K_{r, q}(r) \leq K_{r, r}(r)=1 .
$$

Next we prove that if either $r=2$ or $q<r<2$, then $K_{p, q}(r)=1$ (the idea of the proof here is taken from MARCINKIEWICZ-ZYGMUND [28]).

Let $\left(\omega_{k}\right)$ be an $r$-stable Gaussian sequence. If either $r=2$ or $q<r<\infty$, then

$$
\begin{aligned}
& d_{q}(r)^{q}\left\|\left(\sum_{k=1}^{n}\left|T f_{k}\right|^{r}\right)^{1 / r}\right\|_{q}^{q} \quad \text { [by Lemma 2] } \\
& =\int_{Y} \int_{0}^{1}\left|\sum_{k=1}^{n} T f_{k}(x) \omega_{k}(t)\right|^{q} \mathrm{~d} t \mathrm{~d} v \quad \text { [by Fubini theorem and since } T \text { is linear] } \\
& =\int_{0}^{1}\left\|T\left(\sum_{k=1}^{n} \omega_{k}(t) f_{k}\right)\right\|_{q}^{q} \mathrm{~d} t \\
& \leq\|T\|^{q} \int_{0}^{1}\left\|\sum_{k=1}^{n} \omega_{k}(t) f_{k}\right\|_{q}^{q} \mathrm{~d} t \quad \text { [by boundedness of } T \text { ] } \\
& =\|T\|^{q}\left(\|\left.\int_{X}\left|\sum_{k=1}^{n} \omega_{k}(t) f_{k}(x)\right|^{p} \mathrm{~d} \mu\right|_{L^{q / p}[0,1]}\right)^{q / p} \\
& \leq\|T\|^{q}\left(\int_{X}\left\|\left|\sum_{k=1}^{n} \omega_{k}(t) f_{k}(x)\right|^{p}\right\|_{L^{q / p}[0,1]} \mathrm{d} \mu\right)^{q / p} \\
& =\|T\|^{q}\left\{\int_{X}\left(\int_{0}^{1}\left|\sum_{k=1}^{n} f_{k}(x) \omega_{k}(t)\right|^{q} \mathrm{~d} t\right)^{q / p} \mathrm{~d} \mu\right\}^{q / p} \\
& =d_{q}(r)^{q}\|T\|^{q}\left\{\int_{X}\left(\sum_{k=1}^{n}\left|f_{k}(x)\right|^{r} \mathrm{~d} t\right)^{p / r} \mathrm{~d} \mu\right\}^{q / p}
\end{aligned}
$$$$
\text { [by the Minkowski inequality; } q / p \geq 1 \text { ] }
$$

[by boundedness of $T$ ]

[by Lemma 2]

and thus $K_{p, q}(r) \leq 1$.

Finally, if $2<r<p$, then by using the duality formula of Theorem 1(a) and the above result we obtain

$$
K_{p, q}(r)=K_{q^{\prime}, p^{\prime}}\left(r^{\prime}\right)=1 .
$$

Therefore if $1<p \leq q<\infty$ and $\min (p, 2) \leq r \leq \max (q, 2)$, then $K_{p, q}(r)=1$.

(c) By using Theorem 1(c) and the obvious inequalities we have

$$
K_{p, q}(r) \geq K_{p, q}(2) \geq K_{p, q}^{(2)}(2) \geq K_{l_{2}^{p}, l_{2}^{q}}^{(2)}(2) \geq 1 \text {. }
$$


We conclude that if $K_{p, q}(r)=1$, then $K_{l_{2}^{p}, l_{2}^{q}}^{(2)}(2)=1$, and hence according to Proposition 3(a) either $p \leq 2$ or $q \geq 2$. But in view of Theorem 1 (c) $K_{p, q}(r) \geq K_{p, q}(2) \geq K_{p, q}(2)$, and then $K_{p, q}(r)=1$ implies that $K_{p, q}^{(2)}(2)=1$. Thus from Proposition $3(\mathrm{~b})$ we have

$$
\min \left(p, q^{\prime}\right) \leq r \leq \max \left(p^{\prime}, q\right) \text {. }
$$

(d) Repeating the above arguments and using Proposition 3(c) we obtain the result.

(e) From Proposition 3(a) it only follows that if $q<2<p$, then $K_{p, q}(2) \geq K_{p, q}^{(2)}(2)>1$. However, if $p>q$, then we can construct an operator such that $K_{p, q}^{(2)}(2)>1$.

Example 3. (RIESz [35], p. 493-494). For $\varepsilon>0$ let $T^{\varepsilon}: l_{3}^{p} \rightarrow l_{3}^{q}$ be given by the formula

$$
\begin{aligned}
& T^{\varepsilon}\left(x_{1}, x_{2}, x_{3}\right)=\left(y_{1}, y_{2}, y_{3}\right) \text { with } y_{k}=x_{k}-\varepsilon\left(x_{1}+x_{2}+x_{3}\right), \\
& k=1,2,3
\end{aligned}
$$

For any couple of numbers $(p, q)$ such that $p>q \geq 1$ there exists $\varepsilon>0$ such that $\left\|T^{\varepsilon}\right\|<\left\|T_{C}^{\varepsilon}\right\|$.

Proof. For $x_{1}=1, x_{2}=\exp (2 \pi i / 3)$ and $x_{3}=\exp (-2 \pi i / 3)$ we have $\left|x_{1}\right|=\left|x_{2}\right|=\left|x_{3}\right|$ $=1, x_{1}+x_{2}+x_{3}=0$ and so

$$
\left\|T_{C}^{\varepsilon}\right\| \geq\left(\left|y_{1}\right|^{q}+\left|y_{2}\right|^{q}+\left|y_{3}\right|^{q}\right)^{1 / q} /\left(\left|x_{1}\right|^{p}+\left|x_{2}\right|^{p}+\left|x_{3}\right|^{p}\right)^{1 / p}=3^{1 / q-1 / p} .
$$

On the other hand, let $x_{1}, x_{2}$ and $x_{3}$ be real numbers. Without loss of generality we can assume that $\left|x_{1}\right|^{p}+\left|x_{2}\right|^{p}+\left|x_{3}\right|^{p}=3$. Then we consider the following five cases.

1. If $x_{1}+x_{2}+x_{3}=0$, then

$$
\left|y_{1}\right|^{q}+\left|y_{2}\right|^{q}+\left|y_{3}\right|^{q}=\left|x_{1}\right|^{q}+\left|x_{2}\right|^{q}+\left|x_{3}\right|^{q}<3^{1-q / p}\left(\left|x_{1}\right|^{p}+\left|x_{2}\right|^{p}+\left|x_{3}\right|^{p}\right)^{q / p}=3 .
$$

In the strict inequality we can have equality only if $\left|x_{1}\right|=\left|x_{2}\right|=\left|x_{3}\right|$, but then (since $\left|x_{1}\right|^{p}+\left|x_{2}\right|^{p}+\left|x_{3}\right|^{p}=3$ ) it follows that $\left|x_{1}\right|=\left|x_{2}\right|=\left|x_{3}\right|=1$ and this is impossible according to the equality $x_{1}+x_{2}+x_{3}=0$.

2. If $x_{1}+x_{2}+x_{3} \neq 0$ and $q=1$, then for sufficiently small $\varepsilon>0$ it yields that

$$
\begin{aligned}
& \left|y_{1}\right|+\left|y_{2}\right|+\left|y_{3}\right|=\left|x_{1}-\varepsilon\left(x_{1}+x_{2}+x_{3}\right)\right|+\left|x_{2}-\varepsilon\left(x_{1}+x_{2}+x_{3}\right)\right| \\
& +\left|x_{3}-\varepsilon\left(x_{1}+x_{2}+x_{3}\right)\right| \\
& \leq\left|x_{1}\right|+\left|x_{2}\right|+\left|x_{3}\right|+\varepsilon\left|x_{1}+x_{2}+x_{3}\right|<\varepsilon^{1-1 / p}\left(\left|x_{1}\right|^{p}+\left|x_{2}\right|^{p}+\left|x_{3}\right|^{p}\right)^{1 / p}=3 .
\end{aligned}
$$

3. Let $x_{1}+x_{2}+x_{3} \neq 0$ and $q>1$.

(a) If two of the numbers are equal to zero, for example if $x_{1} \neq 0$ and $x_{2}=x_{3}=0$, then

$$
\begin{aligned}
\left|y_{1}\right|^{q}+\left|y_{2}\right|^{q}+\left|y_{3}\right|^{q} & =\left|x_{1}-\varepsilon x_{1}\right|^{q}+\left|\varepsilon x_{1}\right|^{q}+\left|\varepsilon x_{1}\right|^{q} \\
& =\left|x_{1}\right|^{q}\left[(1-\varepsilon)^{q}+2 \varepsilon^{q}\right] \\
& =3^{q / p}\left[(1-\varepsilon)^{q}+2 \varepsilon^{q}\right]<3
\end{aligned}
$$

for sufficiently small $\varepsilon>0$. 
$(b-c)$ If either one of the numbers is zero, for example $x_{1} \neq 0, x_{2} \neq 0$ and $x_{3}=0$, or all the numbers are different from zero, then for sufficiently small $\varepsilon>0$ we have

$$
\varepsilon\left|x_{1}+x_{2}+x_{3}\right|<\left|x_{k}\right|, \quad k=1,2 \text { or } 3,
$$

and using the binomial series we obtain

$$
\begin{aligned}
&\left|y_{1}\right|^{q}+\left|y_{2}\right|^{q}+\left|y_{3}\right|^{q} \\
&=\left|x_{1}\right|^{q}+\left|x_{2}\right|^{q}+\left|x_{3}\right|^{q}-q \varepsilon\left(\left|x_{1}\right|^{q-1} \operatorname{sgn} x_{1}+\left|x_{2}\right|^{q-1} \operatorname{sgn} x_{2}\right. \\
&\left.\quad+\left|x_{3}\right|^{q-1} \operatorname{sgn} x_{3}\right)\left(x_{1}+x_{2}+x_{3}\right) \\
& \quad+q(q-1) \varepsilon^{2} / 2\left(\left|x_{1}\right|^{q-2}+\left|x_{2}\right|^{q-2}+\left|x_{3}\right|^{q-2}\right)\left(x_{1}+x_{2}+x_{3}\right)^{3}+\ldots \\
&=\left|x_{1}\right|^{q}+\left|x_{2}\right|^{q}+\left|x_{3}\right|^{q}-q \varepsilon\left(\left|x_{1}\right|^{q-1} \operatorname{sgn} x_{1}+\left|x_{2}\right|^{q-1} \operatorname{sgn} x_{2}+\left|x_{3}\right|^{q-1} \operatorname{sgn} x_{3}\right) \\
& \quad \times\left(x_{1}+x_{2}+x_{3}\right)+O\left(\varepsilon^{2}\right) \\
& \leq 3^{1-q / p}\left(\left|x_{1}\right|^{p}+\left|x_{2}\right|^{p}+\left|x_{3}\right|^{p}\right)^{q / p} \\
&-q \varepsilon\left(\left|x_{1}\right|^{q-1} \operatorname{sgn} x_{1}+\left|x_{2}\right|^{q-1} \operatorname{sgn} x_{2}+\left|x_{3}\right|^{q-1} \operatorname{sgn} x_{3}\right) \\
& \times\left(x_{1}+x_{2}+x_{3}\right)+O\left(\varepsilon^{2}\right) \\
&= 3-q \varepsilon\left(\left|x_{1}\right|^{q-1} \operatorname{sgn} x_{1}+\left|x_{2}\right|^{q-1} \operatorname{sgn} x_{2}+\left|x_{3}\right|^{q-1} \operatorname{sgn} x_{3}\right) \\
& \times\left(x_{1}+x_{2}+x_{3}\right)+O\left(\varepsilon^{2}\right)<3 .
\end{aligned}
$$

Thus $\left\|T^{\varepsilon}\right\|<3^{1 / q-1 / p}$ and so $K_{p, q}^{(2)}(2) \geq\left\|T_{C}^{\varepsilon}\right\| /\left\|T^{3}\right\|>1$.

Theorem 1 and Theorem 2 imply immediately the following corollaries.

Corollary 1. (ANDERSEN [1], Figiel-IWANIEC-PelCZYNSKi [9]). If $1 \leq p \leq q \leq \infty$, then $K_{p, q}(2)=1$. In particular, if $p \leq q$, then the norm of the operator $T: L^{p}(\mu) \rightarrow L^{q}(v)$ and the norm of its complexification $T_{C}$ are the same (see also [47], [9], 31]).

Corollary 2. (HERz [14]). Let $1<p<\infty$. Then $K_{p, p}(r)=1$ if, and only if, $\min (p, 2) \leq r \leq \max (p, 2)$.

Corollary 3. If $p>q$, then $K_{p, q}(r)>1$.

Problem 2. Prove or disprove the following conjecture:

Let $1<p \leq q<\infty$. Then $K_{p, q}(r)=1$ if, and only if, $\min (p, 2) \leq r \leq \max (q, 2)$.

Now we will consider the case $p>q$.

Theorem 3. Let $1 \leq q<p<\infty$.

(a) If either $r=2$ or $p<r<2$, then $K_{p, q}(r) \leq d_{p}(r) / d_{q}(r)$.

(b) If $1 \leq q \leq 2$, then $K_{2, q}(2)=d_{2}(2) / d_{q}(2)$.

(c) If $r \neq 2$ and either $q<r \leq \min (p, 2)$ or $\max (q, 2) \leq r<p$, then $K_{p, q}(r)=\infty$. 
Proof. (a) By repeating the proof in Theorem 2(a) we obtain

$$
\begin{aligned}
& d_{q}(r)^{q}\left\|\left(\sum_{k=1}^{n}\left|T f_{k}\right|^{r}\right)^{1 / r}\right\|_{q}^{q} \leq\|T\|^{q} \int_{0}^{1}\left\|\sum_{k=1}^{n} \omega_{k}(t) f_{k}\right\|_{p}^{q} \mathrm{~d} t \\
& \leq\|T\|^{q}\left\{\int_{0}^{1}\left\|\sum_{k=1}^{n} \omega_{k}(t) f_{k}\right\|_{p}^{p} \mathrm{~d} t\right\}^{q / p} \text { the Hölder } \\
& =\|T\|^{q}\left\{\int_{x} \int_{0}^{1}\left|\sum_{k=1}^{n} f_{k}(x) \omega_{k}(t)\right|^{p} \mathrm{~d} t \mathrm{~d} v\right\}^{q / p} \\
& =d_{p}(r)^{q}\|T\|^{q}\left\|\left(\sum_{k=1}^{n}\left|f_{k}\right|^{r}\right)^{1 / r}\right\|_{p}^{p},
\end{aligned}
$$$$
\text { [by the Hölder inequality; } p / q>1 \text { ] }
$$

[by Fubini theorem]

[by Lemma 2]

and thus $K_{p, q}(r) \leq d_{p}(r) / d_{q}(r)$.

(a) It is enough to prove that if $1 \leq q \leq 2$, then

$$
K_{2, q}(2) \geq d_{2}(2) / d_{q}(2) \text {. }
$$

To prove estimate (9) we will construct a concrete operator and then calculate their appropriate norms.

Example 4. Let $1 \leq q \leq 2$ and let $\left(g_{i}\right)$ be a sequence of independent standard Gaussian variables. Consider a linear operator $S: l^{2} \rightarrow L^{q}[0,1]$ defined by $S e_{i}=g_{i}$, for all $i$. Then

$$
\|S\|_{l^{2} \rightarrow L^{q}}=d_{q}(2) \text {, }
$$

and for any $n \in \mathbb{N}$ and all $f_{1}, f_{2}, \ldots, f_{n} \in l^{2}$

$$
\left\|\left(\sum_{k=1}^{n}\left|S f_{k}\right|^{2}\right)^{1 / 2}\right\|_{q} \leq d_{2}(2)\left\|\left(\sum_{k=1}^{n}\left|f_{k}\right|^{2}\right)^{1 / 2}\right\|_{2} \text {. }
$$

In fact, if $f \in l^{2}$, then $f=\sum_{i} c_{i} e_{i}$ and by Lemma 2 we have

$$
\|S f\|_{q}=\left\|\sum_{i} c_{i} S e_{i}\right\|_{q}=\left\|\sum_{i} c_{i} g_{i}\right\|_{q}=d_{q}(2)\left(\sum_{i} c_{i}^{2}\right)^{1 / 2}=d_{q}(2)\|f\|_{2},
$$

i.e., $\|S\|_{l^{2} \rightarrow L^{q}}=d_{q}(2)$.

Furthermore, if $f_{1}, f_{2}, \ldots, f_{n} \in l^{2}$, then $f_{k}=\sum_{i} c_{k, i} e_{i}(k=1,2, \ldots, n)$ and since $\left(g_{i}\right)$ is an orthogonal sequence in $L^{2}[0,1]$, it follows that

$$
\begin{aligned}
& \left\|\left(\left.\sum_{k=1}^{n} S f_{k}\right|^{2}\right)^{1 / 2}\right\|_{q}^{q}=\int_{0}^{1}\left|\left(\left.\sum_{k=1}^{n} S f_{k}(t)\right|^{2}\right)^{1 / 2}\right|^{q} \mathrm{~d} t \\
& =\int_{0}^{1}\left(\sum_{k=1}^{n}\left|\sum_{i} c_{k, i} g_{i}(t)\right|^{2}\right)^{q / 2} \mathrm{~d} t \leq\left(\int_{0}^{1} \sum_{k=1}^{n}\left|\sum_{i} c_{k, i} g_{i}(t)\right|^{2} \mathrm{~d} t\right)^{q / 2}
\end{aligned}
$$


Gasch/Maligranda, Inequalities of the Marcinkiewicz-Zygmund, Herz and Krivine Type

115

$$
\begin{aligned}
& =\left(\sum_{k=1}^{n} \int_{0}^{1}\left|\sum_{i} c_{k, i} g_{i}(t)\right|^{2} \mathrm{~d} t\right)^{q / 2}=\left(d_{2}(2)^{2} \sum_{k=1}^{n} \sum_{i} c_{k, i}^{2}\right)^{q / 2} \\
& =d_{2}(2)^{q}\left(\sum_{i} \sum_{k=1}^{n} c_{k, i}^{2}\right)^{q / 2}=d_{2}(2)^{q}\left\|\left(\sum_{k=1}^{n}\left|f_{k}\right|^{2}\right)^{1 / 2}\right\|_{2}^{q}
\end{aligned}
$$

Therefore

$$
\left\|\left(\sum_{k=1}^{n}\left|S f_{k}\right|^{2}\right)^{1 / 2}\right\|_{q} \leq d_{2}(2)\left\|\left(\sum_{k=1}^{n}\left|f_{k}\right|^{2}\right)^{1 / 2}\right\|_{2} .
$$

Since $g_{1}^{2}, g_{2}^{2}, \ldots$ are i.i.d. (independent identically distributed) random variables with the common mean equal to $d_{2}(2)$, it follows by the Kolmogorov strong law of large numbers that

$$
\frac{1}{n} \sum_{k=1}^{n} g_{k}^{2}(t) \rightarrow d_{2}(2)^{2} \quad \text { a.e. }
$$

and then by the Fatou Lemma we have

$$
\begin{aligned}
& \liminf _{n \rightarrow \infty}\left\|\left(\sum_{k=1}^{n}\left|S e_{k}\right|^{2}\right)^{1 / 2}\right\|_{q} /\left\|\left(\sum_{k=1}^{n}\left|e_{k}\right|^{2}\right)^{1 / 2}\right\|_{2} \\
& =\liminf _{n \rightarrow \infty}\left\|\left(\sum_{k=1}^{n} g_{k}^{2}\right)^{1 / 2}\right\| \|_{q} / n^{1 / 2}=\liminf _{n \rightarrow \infty}\left[\int_{0}^{1}\left(\frac{1}{n} \sum_{k=1}^{n} g_{k}^{2}(t)\right)^{q / 2} \mathrm{~d} t\right]^{1 / 2} \\
& \geq\left[\int_{0}^{1} \liminf _{n \rightarrow \infty}\left(\frac{1}{n} \sum_{k=1}^{n} g_{k}^{2}(t)^{q / 2} \mathrm{~d} t\right)^{1 / q}=\left[\int_{0}^{1}\left(d_{2}(2)^{2}\right)^{q / 2} \mathrm{~d} t\right)\right]^{1 / q}=d_{2}(2) .
\end{aligned}
$$

Thus formula (9) is proved.

(c) We just consider a concrete operator for which it yields $K_{r, q}(r)=\infty$ (concerning the existence of such an operator see Example 5 below). The rest of the cases follows from Theorem 1.

Example 5. Let $1 \leq q<r<2$ and let $\left(g_{i}\right)$ be a sequence of independent $r$-stable Gaussian variables. Consider a linear operator $S: l^{r} \rightarrow L^{q}[0,1]$ defined by $S e_{i}=g_{i}$ for all $i$. Then

$$
\|S\|_{l^{r \rightarrow L^{q}}}=d_{q}(r)
$$

and

$$
\begin{aligned}
& K_{r, q}(r)=\lim _{n \rightarrow \infty} K_{r, q}^{(n)}(r) \\
& \geq \underset{n \rightarrow \infty}{\liminf }\left\|\left(\sum_{k=1}^{n}\left|S e_{k}\right|^{r}\right)^{1 / r}\right\|_{q} /\left[\left\|\left(\sum_{k=1}^{n}\left|e_{k}\right|^{r}\right)^{1 / r}\right\|_{r} d_{q}(r)\right] \\
& =\underset{n \rightarrow \infty}{\liminf }\left\|\left(\sum_{k=1}^{n}\left|g_{k}\right|^{r}\right)^{1 / r}\right\|_{q} /\left[n^{1 / r} d_{q}(r)\right] \\
& =\underset{n \rightarrow \infty}{\liminf }\left[\int_{0}^{1}\left(\frac{1}{n} \sum_{k=1}^{n} \mid g_{k}(t)^{r}\right)^{q / r} \mathrm{~d} t\right]^{1 / q} / d_{q}(r)=\infty,
\end{aligned}
$$

$8^{*}$ 
and so by the Kolmogorov strong law of large numbers we have

$$
\frac{1}{n} \sum_{k=1}^{n}\left|g_{k}(t)\right|^{r} \rightarrow\left\|g_{1}\right\|_{r}=\infty \quad \text { a.e. }
$$

We complete this section by presenting two corollaries and a problem related to Theorem 3 .

Corollary 4 (PISIER [34], Th. 5.4). $K_{2,1}(2)=\sqrt{\frac{\pi}{2}}$.

Corollary 5. If $1 \leq q<p<\infty$, then

$$
K_{p, q}(2) \leq \min \left\{d_{p}(2) / d_{q}(2), d_{q^{\prime}}(2) / d_{p^{\prime}}(2)\right\} .
$$

If either $p=2$ or $q=2$, then equality holds.

Problem 3. Give a complete characterization of the equality $K_{p, q}(r)=\infty$ in terms of $p$, $q$ and $r$.

\section{On the estimates of Krivine type}

KRIVINE [21] proved that $K_{\infty, 1}^{(2)}(2)=\sqrt{2}$. We generalize this result for the case $r \neq 2$. Let us first prove some useful facts about the expression $K_{p, q}^{(2)}(r)$.

Proposition 4. The numbers $K_{p, q}^{(2)}(r)$ are the norms of the function

$$
t_{r}(x, y)=\cos (x-y) /\left[\left(|\sin x|^{r}+|\cos x|^{r^{2}}\right)^{1 / r}\left(|\sin y|^{r^{\prime}}+|\cos y|^{r^{\prime}}\right)^{1 / r^{\prime}}\right]
$$

in $C_{p}([-\pi, \pi]) \hat{\otimes} C_{q^{\prime}}([-\pi, \pi])$.

Proof. By using Proposition 2 we find, as in KrIVIne paper ([21], Prop. 3), that for any representation

$$
\sum_{k=1}^{n} f_{k} \otimes g_{k} \quad\left(f_{k} \text { continuous on } \mathbf{S}_{n}^{r}, g_{k} \text { continuous on } \mathbf{S}_{n}^{r^{\prime}}\right)
$$

of $P_{1} \otimes Q_{1}+P_{2} \otimes Q_{2}$, i.e.,

$$
\sum_{k=1}^{2} P_{k} \otimes Q_{k}(x, y)=x_{1} y_{1}+x_{2} y_{2}=\sum_{k=1}^{n} f_{k} \otimes g_{k},
$$

with $x=\left(\chi_{1}, x_{2}\right) \in \mathbf{S}_{n}^{r}, y=\left(y_{1}, y_{2}\right) \in \mathbf{S}_{n}^{r^{\prime}}$, we can parametrize $x$ and $y$ as follows:

$$
\begin{array}{ll}
x_{1}=\left(|\sin x|^{r}+|\cos x|^{r^{\prime}}\right)^{-1 / r} \cos x, & x_{2}=\left(|\sin x|^{r}+|\cos x|^{r}\right)^{-1 / r} \sin x, \\
y_{1}=\left(|\sin y|^{r^{\prime}}+|\cos y|^{r^{\prime}}\right)^{-1 / r^{\prime}} \cos y, & y_{2}=\left(|\sin y|^{r^{\prime}}+|\cos y|^{r^{\prime}}\right)^{-1 / r^{\prime}} \sin y,
\end{array}
$$

and then we obtain the result. 
We need also

Lemma 4. If $1 \leq r \leq \infty$, then

$$
\max \left(2^{1 / r}, 2^{1-1 / r}\right) \leq K_{\infty, 1}^{(2)}(r) \leq 2 .
$$

Proof. Consider the operator $T: l_{2}^{\infty} \rightarrow l_{2}^{1}$ given by the matrix $\left(\begin{array}{rr}1 & 1 \\ 1 & -1\end{array}\right)$. Then (cf. our Example 1) $\|T\|=2$, and by considering $f_{1}=(0,1)$ and $f_{2}=(1,0)$ we find

$$
K_{\infty, 1}^{(2)}(r) \geq\left(2^{1 / r}+2^{1 / r}\right) / 2=2^{1 / r},
$$

and by considering $f_{1}=(1,1)$ and $f_{2}=(1,-1)$, we obtain

$$
K_{\infty, 1}^{(2)}(r) \geq(2+2) /\left(2^{1+1 / r}\right)=2^{1-1 / r} .
$$

It is easy to prove the second inequality using the inequalities

$$
\max (a, b) \leq\left(a^{r}+b^{r}\right)^{1 / \mathrm{r}} \leq a+b \text { for } a, b \geq 0 .
$$

The main result in this section is the following theorem:

Theorem 4. If $1 \leq r \leq \infty$, then

$$
K_{\infty, 1}^{(2)}(r)=\max \left(2^{1 / r}, 2^{1-1 / r}\right) .
$$

Proof. We already proved in Lemma 4 that $K_{\infty, 1}^{(2)}(r)=2$ in the cases $r=1$ or $r=\infty$. Therefore we assume that $1<r<\infty$. As it was proved by KRIVINE ([21], Th. 1), there exists $f \in C^{\infty}([-\pi, \pi])$ with the following properties.

1) $f$ is an even function and $f(x+\pi)=-f(x)$ for $x \in[-\pi, \pi]$,

2) $f(x)=\sum_{k=0}^{\infty} \alpha_{2 k+1} \cos (2 k+1) x$, where $\alpha_{1}>\sum_{k=1}^{\infty}\left|\alpha_{2 k+1}\right| /(2 k+1)$

3) if $g(x)=\operatorname{sign}(\cos x)$ and $F(x)=\int_{-\pi}^{\pi} f(t-x) g(t) \mathrm{d} t /(2 \pi)=\sum_{k=0}^{\infty} a_{2 k+1} \cos (2 k+1) x$, then

$$
\cos x=\sum_{k=0}^{\infty} b_{2 k+1} F((2 x+1))
$$

where the numbers $b_{2 k+1}$ are obtained by taking the inverse of the Dirichlet series

$$
D(s)=\sum_{k=0}^{\infty} a_{2 k+1}(2 k+1)^{-s}(s \geq 0) \text {, }
$$

4) $\|f\|_{\infty} \sum_{k=0}^{\infty}\left|b_{2 k+1}\right| \leq \sqrt{2}$.

Now since

$$
\min \left(2^{1 / r-1 / 2}, 1\right) \leq\left(|\sin x|^{r}+|\cos x|^{r}\right)^{1 / r} \text { for each } x \in[-\pi, \pi],
$$


it follows that

$$
\begin{aligned}
& \left\|t_{r}(x, y)\right\|_{\otimes} \\
& \leq \sum_{k=0}^{\infty}\left|b_{2 k+1}\right| \| F((2 k+1)(x-y))\left(|\sin x|^{r}+|\cos x|^{r}\right)^{-1 / r} \\
& \quad \times\left(|\sin y|^{r^{\prime}}+|\cos y|^{r^{\prime}}\right)^{-1 / r^{\prime}} \|_{\otimes} \\
& \leq \sum_{k=0}^{\infty}\left|b_{2 k+1}\right|\|f\|_{\infty}\|g\|_{\infty} \max \left(2^{1 / 2-1 / r}, 1\right) \max \left(2^{1 / 2-1 / r^{\prime}}, 1\right) \\
& \leq \max \left(2^{1 / 2-1 / r}, 2^{1 / r-1 / 2}\right)\|f\|_{\infty} \sum_{k=0}^{\infty}\left|b_{2 k+1}\right| \leq \max \left(2^{1-1 / r}, 2^{1 / r}\right) .
\end{aligned}
$$

Hence by using Proposition 3 and Lemma 4 we obtain the desired equality (11).

Problem 4. Calculate $K_{\infty, 1}^{(n)}(r)$ for $n=3,4, \ldots$

KRIVINE [21] proved that $K_{\infty, 1}^{(3)}(2)<1.517$ and $K_{\infty, 1}^{(4)}(2) \leq \pi / 2$.

\section{On the estimates of $K_{p, q}^{(n)}(2)$}

We wish to give the best estimates or, if possible, the exact formulae for the numbers $K_{p, q}^{(n)}(2)$ strictly connected with the complexification constants. The next estimates are our main result in this section.

Theorem 5. If $1 \leq q<p \leq \infty$, then

$$
\max \left\{1,\left(d_{2}^{(n)}\right)^{2} /\left[d_{p^{\prime}}^{(n)} d_{q}^{(n)}\right]\right\} \leq K_{p, q}^{(n)}(2) \leq \min \left\{d_{p}^{(n)} / d_{q}^{(n)}, d_{q^{\prime}}^{(n)} / d_{p^{\prime}}^{(n)}\right\} .
$$

In particular, $K_{\infty, 2}^{(n)}(2)=K_{2,1}^{(n)}(2)=d_{2}^{(n)} / d_{1}^{(n)}$.

Proof. In the same way as in Theorem 2 we can prove, by using Lemma 2 instead of Lemma 3, that

$$
K_{p, q}^{(n)}(2) \leq d_{p}^{(n)} / d_{q}^{(n)} .
$$

From which follows the duality formula $K_{p, q}^{(n)}(2)=K_{p^{\prime}, q^{\prime}}^{(n)}(2) \leq d_{q^{\prime}}^{(n)} / d_{p^{\prime}}^{(n)}$ and the second inequality in (12). For the estimate from below we will construct again an special operator.

Example 6. Let $n \geq 2$ be fixed and let $\left\{\alpha_{k}\right\}_{k=1}^{n}$ be an orthogonal sequence of functions from Lemma 2. Define the operators $T_{n}: L^{p}(0,1) \rightarrow L^{q}(0,1)$ by

$$
T_{n} f(x)=\sum_{k=1}^{n} a_{k} \alpha_{k}(x)
$$

with

$$
a_{k}=n \int_{0}^{1} f(t) \alpha_{k}(t) \mathrm{d} t, \quad k=1,2, \ldots, n, \quad \text { and } \quad f \in L^{p}(0,1)
$$


Then

$$
\left\|T_{n}\right\|_{p, q}=d_{p^{\prime}}^{(n)} d_{q}^{(n)} /\left(d_{2}^{(n)}\right)^{2} .
$$

Proof. Since

$$
\left\|T_{n} f\right\|_{q}=d_{q}^{(n)}\left(\sum_{k=1}^{n} a_{k}^{2}\right)^{1 / 2}=d_{p}^{(n)} / d_{2}^{(n)}\left\|T_{n} f\right\|_{2},
$$

it follows that

$$
\left\|T_{n}\right\|_{p, q}=d_{q}^{(n)} / d_{2}^{(n)}\left\|T_{n}\right\|_{p, 2} .
$$

But $\left\|T_{n}\right\|_{p, 2}=\left\|T_{n}^{*}\right\|_{2, p^{\prime}}$, where the conjugate operator is given by

$$
T_{n}^{*} g(x)=\sum_{k=1}^{n} b_{k} \alpha_{k}(x)
$$

where

$$
b_{k}=n \int_{0}^{1} g(t) \alpha_{k}(t) \mathrm{d} t, \quad k=1,2, \ldots, n .
$$

But Parseval's equality implies that $\left\|T_{n}^{*}\right\|_{2, p}$, is attained if the function is of the form

$$
g(x)=\sum_{k=1}^{n} c_{k} \alpha_{k}(x)
$$

Since the $L^{2}(0,1)$ norm of this function is equal to $d_{2}^{(n)}\left(\sum_{k=1}^{n} c_{k}\right)^{1 / 2}$, it follows that

$$
\left\|T_{n}\right\|_{p, 2}=\left\|T_{n}^{*}\right\|_{2, p^{\prime}}=d_{p^{\prime}}^{(n)} / d_{2}^{(n)}
$$

and hence (13) is proved.

Since

$$
T_{n}\left(\alpha_{k}\right)=\alpha_{k} \text { and } \sum_{k=1}^{n} \alpha_{k}^{2}=1
$$

it follows that

$$
\left\|\left(\sum_{k=1}^{n}\left|T_{n}\left(\alpha_{k}\right)\right|^{2}\right)^{1 / 2}\right\|_{q}=\left\|\left(\sum_{k=1}^{n}\left|\alpha_{k}\right|^{2}\right)^{1 / 2}\right\|_{p}=1
$$

and so

$$
\begin{aligned}
K_{p, q}^{(n)}(2) & \geq\left\|\left(\sum_{k=1}^{n}\left|T_{n}\left(\alpha_{k}\right)\right|^{2}\right)^{1 / 2}\right\| /\left[\left\|\left(\sum_{k=1}^{n}\left|\alpha_{k}\right|^{2}\right)^{1 / 2}\right\|_{p}\left\|T_{n}\right\|_{p, q}\right] \\
& =1 /\left\|T_{n}\right\|_{p, q}=\left(d_{2}^{(n)}\right)^{2} /\left[d_{p^{\prime}}^{(n)}, d_{q}^{(n)}\right] .
\end{aligned}
$$

These estimates also prove the first inequality in (12).

Proposition 5. Let $p>q \geq 1$. If $1 / p+1 / q \geq 1$, then $\min \left\{d_{p}^{(n)} / d_{q}^{(n)}, d_{q^{\prime}}^{(n)} / d_{p^{\prime}}^{(n)}\right\}=d_{p}^{(n)} / d_{q}^{(n)}$, and if $1 / p+1 / q<1$, then $\min \left\{d_{p}^{(n)} / d_{q}^{(n)}, d_{q^{\prime}}^{(n)} / d_{p^{\prime}}^{(n)}\right\}=d_{q^{\prime}}^{(n)} / d_{p^{\prime}}^{(n)}$. 
Proof. According to (13) we have

$$
\left\|T_{n}\right\|_{p}:=\left\|T_{n}\right\|_{p, p}=d_{p^{\prime}}^{(n)} d_{p}^{(n)} /\left(d_{2}^{(n)}\right)^{2} .
$$

If $1 / p+1 / q \geq 1$, then $q<p \leq q^{\prime}$ and, by using the Riesz-Thorin interpolation theorem (see [2] or [3]), we find

$$
\left\|T_{n}\right\|_{p} \leq\left\|T_{n}\right\|_{q}^{\theta}\left\|T_{n}\right\|_{q^{\prime}}^{1-\theta}=\left\|T_{n}\right\|_{q} .
$$

This means that

$$
d_{q^{\prime}}^{(n)} / d_{p^{\prime}}^{(n)} \geq d_{p}^{(n)} / d_{q}^{(n)} .
$$

In the case $1 / p+1 / q<1$ the proof is similar.

In the special case $n=2$ the estimates (12) are particularly interesting in the complexification problem. Recall that in this case the constant $\gamma$ in the complexification procedure is equal to the constant $K_{L^{p}(\mu), L^{q}(v)}^{(2)}(2)$. Therefore, according to Theorem 2, Theorem 4 and Theorem 5 , we get immediately the following results about the constant $\gamma$ and $K_{p, q}^{(2)}(2)$ $=\sup _{\mu, v} \gamma\left(L^{p}(\mu), L^{q}(v)\right)$.

Corollary 6. The following relations hold:

(a) $1 \leq \gamma\left(L^{p}(\mu), L^{q}(v)\right) \leq \sup _{\mu, v} \gamma\left(L^{\infty}(\mu), L^{1}(v)\right)=K_{\infty, 1}^{(2)}(2)=\sqrt{2}$.

(b) If $1 \leq p \leq q \leq \infty$, then $\gamma\left(L^{p}(\mu), L^{q}(v)\right)=1$ for arbitrary $\sigma$-finite measures $\mu$ and $\nu$.

(c) If $1 \leq q<p<\infty$, then

$$
\max \left(1,\left[d_{2}^{(2)}\right]^{2} /\left[d_{p^{\prime}}^{(2)} d_{q}^{(2)}\right]\right) \leq K_{p, q}^{(2)}(2) \leq \min \left(d_{p}^{(2)} / d_{q}^{(2)}, d_{q^{\prime}}^{(2)} d_{p^{\prime}}^{(2)}\right)
$$

In particular $K_{\infty, 2}^{(2)}(2)=\frac{\pi}{2 \sqrt{2}}$.

In [43] it was shown that if $1 \leq p \leq q \leq \infty$, then $\gamma\left(l_{n}^{p}, l_{n}^{q}\right)=1$. By using the equality (1), ZYGMUND [50, p. 181] proved that $\gamma\left(L^{p}(\mu), L^{p}(v)\right)=1$. Moreover, by using an idea of ZYGMUND, SEREDA-VERBICKII [47, Th. 1] proved that $\gamma\left(L^{p}(\mu), L^{q}(v)\right)=1$ for arbitrary $\sigma$-finite measures $\mu$ and $v$, provided that $1 \leq p \leq q \leq \infty$. Formula (14) was also proved by VERBICKII [46].

Remark 2. The constant $\gamma$ depends essentially on the measures $\mu$ and $\nu$. For example, $\gamma\left(l_{2}^{p}, l_{2}^{q}\right)=1$ if, and only if, either $p \leq 2$ or $2 \leq q$ (cf. Proposition 3(a)). GaSCH [11] proved that $\gamma\left(l_{2}^{p}, l_{2}^{q}\right)=\gamma\left(l_{2}^{\infty}, l_{2}^{p^{\prime}}\right)=\max \left(1,2^{1-p^{\prime} / 2}\right)$.

Problem 5. Let $1 \leq q<p<\infty$. Compute $\gamma\left(l_{m}^{p}, 1_{n}^{q}\right)$ for any $m, n \geq 2$ and also $\gamma\left(l^{p}, l^{q}\right)$.

\section{On some estimates in Banach lattices}

We now make some general observation regarding the constants $K$ where general Banach lattices $X$ instead of the Lebesgue spaces $L^{p}$ are considered. 
Let $n \in \mathbb{N}$ and $1 \leq r \leq \infty$. For any real Banach lattices $X, Y$ and any continuous linear operator $T: X \rightarrow Y$ let us denote

$$
\begin{gathered}
K_{X, Y}^{(n)}(r)=\inf \left\{C \geq 1:\left\|\left(\sum_{k=1}^{n}\left|T x_{k}\right|^{r}\right)^{1 / r}\right\|_{Y} \leq C\|T\|\left\|\left(\sum_{k=1}^{n}\left|x_{k}\right|^{r}\right)^{1 / r}\right\|_{X}\right. \\
\left.\quad \text { for all } x_{1}, \ldots, x_{n} \in X \text { and any above } T\right\},
\end{gathered}
$$

and we write $K_{X, X}^{(n)}(r)=K_{X}^{(n)}(r)$.

Theorem 6. (a) The following equality holds:

$$
\sup \left\{K_{X, Y}^{(n)}(r): X, Y \text { Banach lattices }\right\}=K_{\infty, 1}^{(n)}(r) .
$$

(b) If $X, Y$ are Banach function spaces and $Y^{\prime \prime} \equiv Y$, then $K_{X, L^{\infty}}^{(n)}(2)=K_{L^{1}, Y}^{(n)}(2)=1$.

(c) There exists a two-dimensional symmetric space $X$ for which $K_{X}^{(2)}(2)>1$.

Proof. (a) The proof in a general case of Banach lattices $X, Y$ can be found in [19] or [25]. Here we include a proof in the case of Banach function spaces $X, Y$ with $Y^{\prime \prime} \equiv Y$ (see also [34]).

For $x_{1}, \ldots, x_{n} \in X(\mu)$ let us put

$$
\psi=\left(\sum_{k=1}^{n}\left|x_{k}\right|^{r}\right)^{1 / r} \text { and } y_{k}=x_{k} / \psi
$$

(with the convention $0 / 0=0$ ), $k=1,2, \ldots, n$. Clearly, $y_{k} \in L^{\infty}(\mu)$.

Define $M: L^{\infty}(\mu) \rightarrow X(\mu)$ as a multiplication by the function $\psi$, i.e., $M x=x \cdot \psi$. Then

$$
\|M\|_{L^{\infty} \rightarrow X} \leq\|\psi\|_{X}, \quad M y_{k}=x_{k} \text { and }\left\|\left(\sum_{k=1}^{n}\left|y_{k}\right|^{r}\right)^{1 / r}\right\|_{X} \leq 1 .
$$

Let $\varphi$ by an arbitrary positive function $\varphi \in Y^{\prime}$ with $\|\varphi\|_{Y^{\prime}} \leq 1$. Define $N: Y(v) \rightarrow L^{1}(v)$ as a multiplication operator by $\varphi$, i.e., $N y=y \cdot \varphi$.

Consider now the operator NTM: $L^{\infty}(\mu) \rightarrow L^{1}(v)$. For such an operator we have

$$
\begin{aligned}
& \left\|\left(\sum_{k=1}^{n}\left|N T M y_{k}\right|^{r}\right)^{1 / r}\right\|_{L^{1}} \leq K_{\infty, 1}^{(n)}(r)\|N T M\|_{L^{\infty} \rightarrow L^{1}}\left\|\left(\sum_{k=1}^{n}\left|y_{k}\right|^{r}\right)^{1 / r}\right\|_{L^{\infty}} \\
& \leq K_{\infty, 1}^{(n)}(r)\|N\|_{Y \rightarrow L^{1}}\|T M\|_{L^{\infty} \rightarrow Y} \leq K_{\infty, 1}^{(n)}(r)\|T M\|_{L^{\infty}} \\
& \leq K_{\infty, 1}^{(n)}(r)\|T\|_{X \rightarrow Y}\|M\|_{L^{\infty} \rightarrow X} \leq K_{\infty, 1}^{(n)}(r)\|T\|_{X \rightarrow Y}\|\psi\|_{X} .
\end{aligned}
$$

Since $N T M\left(y_{k}\right)=N T\left(x_{k}\right) \cdot \varphi$, it follows

$$
\int\left(\sum_{k=1}^{n}\left|T x_{k}\right|^{r}\right)^{1 / r} \cdot \varphi \mathrm{d} v \leq K_{\infty, 1}^{(n)}(r)\|T\|_{X \rightarrow Y}\|\psi\|_{X} .
$$

Taking the supremum over all above $\varphi$ we obtain

and so $K_{X, Y}^{(n)}(r) \leq K_{\infty, 1}^{(n)}(r)$.

$$
\begin{aligned}
\left\|\left(\sum_{k=1}^{n}\left|T x_{k}\right|^{r}\right)^{1 / r}\right\|_{Y} & =\left\|\left(\sum_{k=1}^{n}\left|T x_{k}\right|^{r}\right)^{1 / r}\right\|_{Y^{\prime \prime}} \leq K_{\infty, 1}^{(n)}(r)\|T\|_{X \rightarrow Y}\|\psi\|_{X} \\
& =K_{\infty, 1}^{(n)}(r)\|T\|_{X \rightarrow Y}\left\|\left(\sum_{k=1}^{n}\left|x_{k}\right|^{r}\right)^{1 / r}\right\|_{X},
\end{aligned}
$$


(b) If $T: X \rightarrow L^{\infty}(v)$ is a bounded operator, then, by using Lemma 1 twice with $p=\infty$, we obtain for all $f_{1}, \ldots, f_{n} \in X$

$$
\begin{aligned}
\left\|\left(\sum_{k=1}^{n}\left|T f_{k}\right|^{2}\right)^{1 / 2}\right\|_{L^{\infty}(v)} & =\|\| \sum_{k=1}^{n} T f_{k} \alpha_{k}(t)\left\|_{L^{\infty}(0,1)}\right\|_{L^{\infty}(v)} \\
& =\|\| T\left(\sum_{k=1}^{n} f_{k} \alpha_{k}(t)\right)\left\|_{L^{\infty}(v)}\right\|_{L^{\infty}(0,1)} \\
& \leq\|T\|_{X \rightarrow L^{\infty}}\|\| \sum_{k=1}^{n} f_{k} \alpha_{k}(t)\|\|_{X} \|_{L^{\infty}(0,1)} \\
& \leq\|T\|_{X \rightarrow L^{\infty}}\|\| \sum_{k=1}^{n} f_{k} \alpha_{k}(t)\|\|_{L^{\infty}(0,1)} \|_{X} \\
& =\|T\|_{X \rightarrow L^{\infty}} \|\left(\sum_{k=1}^{n}\left|f_{k}\right|^{2}\right)_{X}^{1 / 2},
\end{aligned}
$$

and so $K_{X, L^{\infty}}^{(n)}(2)=1$.

Now if $T: L^{1}(\mu) \rightarrow Y$ is a bounded operator, then again using Lemma 1 twice with $p=1$, the generalized Minkowski inequality (cf. [18], p. 45) and Fubini theorem, we get

$$
\begin{aligned}
d_{1}^{(n)}\left\|\left(\sum_{k=1}^{n}\left|T f_{k}\right|^{2}\right)^{1 / 2}\right\| & =\left\|\sum_{k=1}^{n} T f_{k} \alpha_{k}(t)\right\|_{L^{1}(0,1)} \|_{Y} \\
\leq & \|\| \sum_{k=1}^{n} T f_{k} \alpha_{k}(t)\left\|_{Y}\right\|_{L^{1}(0,1)} \leq\|T\|_{L^{1 \rightarrow Y}} \\
& \times\|\| \sum_{k=1}^{n} f_{k} \alpha_{k}(t)\|\|_{L^{1}(t)}\|\|_{L^{1}(0,1)} \\
= & \|T\|_{L^{1} \rightarrow Y}\|\| \sum_{k=1}^{n} f_{k} \alpha_{k}(t)\|\|_{L^{1}(0,1)} \|_{L^{1}(\mu)} \\
= & d_{1}^{(n)}\|T\|_{L^{1} \rightarrow Y}\left\|\left(\sum_{k=1}^{n}\left|f_{k}\right|^{r}\right)^{1 / r}\right\|_{X} .
\end{aligned}
$$

for all $f_{1}, \ldots, f_{n} \in L^{1}(\mu)$.

Therefore $K_{L^{1}, Y}^{(n)}(2)=1$.

(c) It is proved by presenting the following two-dimensional example:

Example 7. (see also [40]). The two-dimensional space $X=\mathbb{R}^{2}$ with the norm of $x=\left(x_{1}, x_{2}\right)$ given by

$$
\|x\|=\left|x_{1}\right|+\left|x_{2}\right|+\sqrt{2} \max \left(\left|x_{1}\right|,\left|x_{2}\right|\right)
$$

is a symmetric space. For the operator

$$
T: X \rightarrow X \quad \text { defined by } \quad T=\left(\begin{array}{rr}
1 & 1 \\
1 & -1
\end{array}\right)
$$


we have the inequalities

$$
\begin{aligned}
\|T x\| & \leq\left|x_{1}+x_{2}\right|+\left|x_{1}-x_{2}\right|+\sqrt{2} \max \left(\left|x_{1}+x_{2}\right|,\left|x_{1}-x_{2}\right|\right) \\
& \leq 2 \max \left(\left|x_{1}\right|,\left|x_{2}\right|\right)+\sqrt{2}\left(\left|x_{1}\right|+\left|x_{2}\right|\right)=\sqrt{2}\|x\|
\end{aligned}
$$

with the equality for $x=(1,0)$ or $x=\left(x_{1}, x_{1}\right)$, i.e., $\|T\|=\sqrt{2}$.

On the other hand, for $x_{0}=(1,1+i)$ we obtain

$$
\left\|T_{C} x_{0}\right\| /\left\|x_{0}\right\|=[(\sqrt{2}+1) \sqrt{5}+1] /(3+\sqrt{2})>\sqrt{2},
$$

which means that $\|T\|<\left\|T_{C}\right\|$ and so $K_{X}^{(2)}(2)>1$.

We remark that an example of a four-dimensional non-symmetric space $X$ for which $K_{X}^{(2)}(2)>1$ was given by Verbickil-SEREDA [47]. Namely, if $X=l_{2}^{1} \oplus l_{2}^{\infty}$ is the direct sum of the two-dimensional spaces $l_{2}^{1}$ and $l_{2}^{\infty}$ with the norm of $x=\left(x_{1}, x_{2}, x_{3}, x_{4}\right)$ equal to

$$
\|x\|=\left|x_{1}\right|+\left|x_{2}\right|+\max \left(\left|x_{3}\right|,\left|x_{4}\right|\right),
$$

and if operator $S: X \rightarrow X$ is given by

$$
S=\left(\begin{array}{ll}
0 & T \\
0 & 0
\end{array}\right), \quad \text { where } \quad T=\left(\begin{array}{rr}
1 & 1 \\
1 & -1
\end{array}\right)
$$

then

$$
\|S\|_{X \rightarrow X}=\|T\|_{\infty, 1}=2, \quad\left\|S_{C}\right\|_{X_{C} \rightarrow X_{C}}=\left\|T_{C}\right\|_{\infty, 1}=2 \sqrt{2}
$$

and thus

$$
K_{X}^{(2)}(2) \geq\left\|S_{C}\right\|_{X_{C} \rightarrow X_{C}} /\|S\|_{X \rightarrow X}=\sqrt{2} .
$$

Finally we present a corollary of Theorem 6(a) and a problem connected to Theorem 6(c).

Corollary 7. For arbitrary Banach lattices $X$ and $Y$

$$
K_{X, Y}^{(2)}(r) \leq K_{\infty, 1}^{(2)}(r)=\max \left(2^{1-1 / r}, 2^{1 / r}\right)
$$

Problem 6. Characterize the Banach lattices $X$ for which $K_{X}^{(2)}(2)=1$.

Remark 3. If we take finite dimensional subspaces $X_{m} \subset L^{p}$ and $Y_{n} \subset \mathrm{L}^{q}$, then it is not true in general that

$$
\lim _{m, n \rightarrow \infty} K_{X_{m}, Y_{n}}^{(2)}(2)=K_{p, q}^{(2)}(2) .
$$

VERBICKII [46] proved that if $X_{m, p}$ are subspaces of $L^{p}(0,2 \pi)$ of all trigonometric polynomials of degree $m$ with the $L^{p}$-norm and similarly $X_{n, q}$ are subspaces of $L^{q}(0,2 \pi)$ of all trigonometric polynomials of degree $n$ with the $L^{q}$-norm, then for $p>q \geq 1$ and $1 / p+1 / q<1$

$$
K_{X_{m}, Y_{n}}^{(2)}(2)=d_{p}^{(2)} / d_{q}^{(2)}>d_{q^{\prime}}^{(2)} / d_{p^{\prime}}^{(2)}=K_{p, q}^{(2)}(2)
$$

holds. 


\section{Some additional results and remarks}

\subsection{The complex case}

\subsubsection{The constants in the complex case}

If $L^{p}(\mu)$ and $L^{q}(v)$ are spaces over the complex field $\mathbb{C}$, then equalities (2) and (3) hold but with different constants (cf. [16] or [32], p. 289 and p. 299, respectively):

$$
\begin{aligned}
& d_{p, C}^{(n)}=[\Gamma((p+2) / 2) \Gamma(n) /(\Gamma((p+2 n) / 2))]^{1 / p} \\
& d_{p, c}(r)=2[\Gamma(1-p / r) \Gamma((p+2) / 2) / \Gamma((1-p / 2))]^{1 / p} .
\end{aligned}
$$

Therefore all our results where we have used the equalities (2) or (3) can be extended to the complex spaces. For example, in the complex case we have then

$$
K_{2,1}^{C}(2)=\frac{2}{\sqrt{\pi}}
$$

(in the real case it was $K_{2,1}(2)=\sqrt{\frac{\pi}{2}}$, cf. Corollary 4 ).

\subsubsection{The Grothendieck constants in the real and the complex case}

The Grothendieck inequality has important applications in the theory of Banach spaces (cf. [12], [19], [24], [25], [31], [34]). Let us note that in our notations it means

$$
K_{\infty, 1}(2)=K_{G}:=\lim _{n \rightarrow \infty} K_{G}(n)=\lim _{n \rightarrow \infty} K_{\infty, 1}^{(n)}(2)
$$

and

$$
K_{\infty, 1}^{C}(2)=K_{G}^{C}:=\lim _{n \rightarrow \infty} K_{G}^{C} \mp \lim _{n \rightarrow \infty} K_{\infty, 1}^{(n), C}(2)
$$

The above complex constants are always smaller than the real ones. The exact values of $K_{G}$ and $K_{G}^{C}$ are not known although the study about these constants is intense (cf. [13] and [31]): Grothendieck (1956), Kaijser (1973), Rietz (1974), Maurey (1974), Pisier (1978), Krivine (1979), Davie (1984), HaAgerup (1987), KöNIG (1990). The best known upper estimates for the Grothendieck constant $K_{G}$ and $K_{G}^{C}$ are due to KRIVINE [21]:

$$
K_{G} \leq \frac{\pi}{2 \ln (1+\sqrt{2})} \approx 1.782
$$

and HAAGERUP [13]:

$$
K_{G}^{C} \leq \frac{8}{\pi\left(k_{0}+1\right)} \approx 1.405,
$$


where $k_{0}$ is the solution of a certain equation involving elliptic integrals. The upper estimate for $K_{G}$ is conjectured to be the exact value by KRIVINE, whereas the bound for $K_{G}^{c}$ by HAAGERUP is not considered to be precise.

Very little is known on the estimates from below. The best known of these estimates are $K_{G}>\pi / 2$ (announced by KRIVINE [21] and unpublished of DAVIE - proof cf. [17]) and $K_{G}^{\mathrm{C}}>1.338$ (in particular $K_{G}^{C}>4 / \pi$ ). From our estimate (12) we can get that $K_{G} \geq \pi / 2$ which was already known by Grothendieck.

The real constants $K_{G}(n)$ have been estimated by KRIVINE [21], using the spherical function technique (cf. our Sections 2 and 4). TONGE [45], started to estimate the numbers $K_{G}^{C}(n)$ for $n=2$ and 3 . He obtained $K_{G}^{C}(2) \leq 2 / \sqrt{2} \approx 1.213$ and $K_{G}^{C}(2) \leq 3 / 2$. After that KöNIG [17], by modifying and combining techniques of KRIVINE [21] and HAAGERUP [13], gave better estimates from above and also estimates from below for the constants $K_{G}^{C}(n)$.

\subsubsection{An interpolation proof of a result of HERz in the complex case}

If

$$
T: L^{p}(\mu) \rightarrow L^{q}(v), \quad 1 \leq p<\infty,
$$

is a bounded operator, then the natural extension

$$
T_{E}: L^{q}\left(l_{n}^{r}\right) \rightarrow L^{p}\left(l_{n}^{r}\right)
$$

is also bounded for $r=2$ [using the equality (3) in the complex case] and $r=p$. Using the complex interpolation method ( $c$ f. [2], Th. 5.1.2) we have

$$
\left[L^{p}\left(l_{n}^{2}\right), L^{p}\left(l_{n}^{p}\right)\right]_{\theta}=L^{p}\left(l_{n}^{r}\right)
$$

with equality of the norms, when $1 / r=(1-\theta) / 2+\theta / p, 0 \leq \theta \leq 1$, and so

$$
\begin{aligned}
\left\|T_{E}\right\|_{L^{p}\left(l_{n}^{r}\right) \rightarrow L^{p}\left(l_{n}^{r}\right)} & \leq\left(\left\|T_{E}\right\|_{L^{p}\left(l_{n}^{2}\right) \rightarrow L^{p}\left(l_{n}^{2}\right)}\right)^{1-\theta}\left(\left\|T_{E}\right\|_{L^{p}\left(l_{n}^{p}\right) \rightarrow L^{p}\left(l_{n}^{p}\right)}\right)^{\theta} \\
& \leq\left(\|T\|_{p, p}\right)^{1-\theta}\left(\|T\|_{p, p}\right)^{\theta}=\|T\|_{p, p} .
\end{aligned}
$$

Therefore we get our Corollary 1:

$$
\text { If } \min (p, 2) \leq r \leq \max (p, 2) \text {, then } K_{p, p}(r)=1 .
$$

This possibility of the proof was suggested also by HERZ-RIVIERE [15], p. 512 and MCCARTHY [29], p. 210.

\subsection{The $B$-valued extensions of operators}

The estimates discussed above suggests the following general scheme. For a given Banach space $B$ and for any bounded linear operator

$$
T: L^{p}(\mu) \rightarrow L^{q}(v)
$$

we denote by

$T \otimes I: L^{p}(\mu) \otimes B \rightarrow L^{q}(v) \otimes B$ 
the extension which on $B$-valued function of the form

$$
g=\sum_{i=1}^{n} f_{i}(x) b_{i}, \quad f_{i} \in L^{p}(\mu), \quad b_{i} \in B \quad(i=1,2, \ldots, n, n=1,2, \ldots)
$$

is given by:

$$
T \otimes I\left(\sum_{i=1}^{n} f_{i}(x) b_{i}\right)=\sum_{i=1}^{n} T f_{i}(x) b_{i} .
$$

If this operator $T \otimes I$ is bounded from $L^{p}(\mu, B)$ into $L^{q}(v, B)$, then it has an extension on all $L^{p}(\mu, B)$ by continuity; denote this extension by $T^{B}$. We are looking for Banach spaces $B$ such that, this operator $T \otimes I$ has a $B$-valued bounded extension $T^{B}: L^{p}(\mu, B)$ $\rightarrow L^{q}(v, B)$ and $\left\|T^{B}\right\| \leq C_{p, q, B}\|T\|$.

This question was considered and solved by HERz [14], KWAPIEN [22] and RUBIo DE Francia-TorRea [39] (see also [10]), but nothing is said about the constant $C_{p, q, B}$. If we know, additionally, that $T$ is a positive operator, then for any Banach space $B, T \otimes I$ has a $B$-valued extension $T^{B}$ and the norms are the same $\left\|T^{B}\right\|=\|T\|$ (cf. [36], Th. 1 or [10], Th. 1.12).

\subsection{Estimates in other spaces}

\subsubsection{A more general version of the Marcinkiewicz-Zygmund theorem}

The same proof as in the MARCINKIEWICZ-ZYGmund paper [28] (cf. also [8], p. 203-204) giving a more general version of the Marcinkiewicz-Zygmund theorem:

If $1 \leq p<\infty$ and $X$ is a closed subspace of $L^{p}(\mu)$, then $K_{X, L^{p}(v)}^{(n)}(2)=1$ for all $n \in \mathbb{N}$.

Our proof of Theorem 2(b) establishes even more (cf. also [9], Th. 1.1):

$$
\text { If } 1 \leq p \leq q \leq \infty \text { and } X \text { is a closed subspace of } L^{p}(\mu) \text {, then } K_{X, L^{q}(v)}^{(n)}(2)=1 \text { for all } n \in \mathbb{N} \text {. }
$$

\subsubsection{Weak type inequalities}

We can consider also operators from $L^{p}(\mu)$-space into a bigger space than the $L^{q}(v)$ space, namely the so called weak- $L^{q}$ space or Marcinkiewicz space $L^{q \infty}$; such operators are called of weak type $(p, q)$. Since the weak- $L^{q}$ norm of a function has an equivalent form

$$
\|f\|_{q \infty} \approx \sup _{v(A)<\infty} v(A)^{1 / q-1 / s}\left\|f \chi_{A}\right\|_{s}, \quad 0<s<q \leq \infty,
$$

as was observed first by CotLaR [5], Th. 2a (see also [36], p. 652), can be used to prove results about strong type operators. However, in this case we have no control over the constants. Such results for the weak-operators were given by OBERLIN [30] and RUBio DE Francia [36] (see also [10], Th. 2.9). 


\subsubsection{Results for other spaces}

If $X$ is a Banach function space with the Fatou norm and $1 \leq q \leq \infty$, then $X^{(q)}$ is the space of all measurable $f$ for which $|f|^{q} \in X$, with the norm

$$
\|f\|_{X^{(q)}}=\left\||f|^{q}\right\|_{X}^{1 / q} .
$$

We can consider the case of real operators $T: L^{p}(\mu) \rightarrow X^{(q)}$ and we can prove, in the same way as in Theorem 2 , that if either $r=2$ or $1 \leq p<r<2$, then

$$
\left\|\left(\sum_{k=1}^{n}\left|T f_{k}\right|^{r}\right)^{1 / r}\right\|_{X^{(q)}} \leq d_{p}(r) / d_{\min (p, q)}(r)\left\|\left(\sum_{k=1}^{n}\left|f_{k}\right|^{r}\right)^{1 / r}\right\|_{p}
$$

(see also [1]). This generalization contains both strong $(p, q)$-type operators and weak $(p, q)$-type operators.

Some results for operators between spaces of functions with finite variation, spaces of continuous functions and $C^{*}$-algebras were obtained by ZYGMUND [49], VERBICKII-SEREDA [47] and PISIER [33].

Note 1. On the occasion of Professor Mischa CoTLAR seventy-fifth birthday a special session was organized in his honor at the Universidad de Buenos Aires, Argentina, on April 18, 1988. Mischa Cotlar's bibliography, talks by A. P. Calderón, J. L. Massera, M. SADOSKY about his work and a collection of 34 papers dedicated to him were presented. A list of publications and a survey of his contributions in mathematics, written by J. HoRvÁtH and C. SADOSKY are published in "Analysis and Partial Differential Equations", Edited by C. Sadosky, Marcel Dekker, New York and Basel 1990, xxvii + 742 pp.

Note 2. The results of Sections 2 and 4 were presented by the first named author on the XXXIX Anual Convención Anual AsoVAC held at Caracas - Venezuela, November 22, 1989 and XL Anual Convención AsoVAC held at Cumaná - Venezuela, November 20, 1990. The results of Sections $2-5$ were presented by the second named author on The $21^{\text {st }}$ Nordic Congress of Mathematicians held at Luleå - Sweden, June 8-12, 1992 and on The $3^{\text {rd }}$ International Conference on Function Spaces held at Poznan - Poland, Aug. 30-Sept. 4, 1992.

Note 3 (added in May 1993). Referee of this paper pointed out that A. DeFanT, in the forthcoming paper "Best constants for the norm of the complexification of operators between $L_{p}$-spaces", proved the following result:

If either $1 \leq q \leq p \leq 2$ or $2 \leq q \leq p \leq \infty$, then $K_{p, q}^{(2)}(2)=d_{p}^{(2)} / d_{q}^{(2)}$.

\section{References}

[1] K. F. ANDERSEN, Inequalities of Scalar-valued Linear Operators that Extend to their Vector-valued Analogues, J. Math. Anal. Appl. 77 (1980), 264-269

[2] J. BERGH and LÖFSTROM, Interpolation Spaces. An introduction, Springer-Verlag 1976

[3] Yu. A. BrudnYI and N. Ya. KRUGLJAK, Interpolation Functors and Interpolation Spaces I, North-Holland, Amsterdam 1991

[4] A. V. Bukhvalov, A. I. Veksler and G. Ya. Lozanovski, Banach Lattices - some Banach Aspects of their Theory, Uspekhi Mat. Nauk 34 (1979), 137-183; English transl. Russian Math. Surveys 34 (1979), 159-212 
[5] M. CotLAR, A General Interpolation Theorem for Linear Operations, Rev. Mat. Cuyana 1 (1955), $57-84$

[6] M. CotLaR and C. SADOSKY, Vector-valued Inequalities of Marcinkiewicz-Zygmund and Grothendieck Type for Generalized Toeplitz Kernels, Lectore Notes in Math. 992, 278-308 (1983)

[7] E. Dubinsky, A. Pelczynski and H. P. Rosenthal, On Banach Spaces for which $\Pi_{2}\left(L_{\infty}, X\right)$ $=B\left(L_{\infty}, X\right)$, Studia Math. $46(1972), 617-648$

[8] R. E. Edwards and G. I. Gaudry, Littlewood-Paley and Multiplier Theory, Springer-Verlag 1977

[9] T. Figiel, T. IWANiec and A. Pelczynski, Computing Norms and Critical Exponents of some Operators in $L^{p}$ Spaces, Studia Math. 79 (1984), 227-274

[10] J. Garcia-Cuerva and J. L. Rubio de Francia, Weighted Norm Inequalities and Related Topics, North-Holland, Amsterdam 1985

[11] J. Gasch, Constantes Absolutas en la Complejificación de los Espacios lp con Dominio Bidimensional, Manuscript, Universidad Central de Venezuela, November 1990, 22 pp.

[12] J. E. GILbERT and T. J. LeIH, Factorization, Tensor Products, and Bilinear Forms in Banach Space Theory, Manuscript, University of Texas at Austin 1978, $123 \mathrm{pp}$.

[13] U. Hangerup, A New Upper Bound for the Complex Grothendieck Constant, Israel J. Math. 60 (1987), 199-224

[14] C. Herz, The Theory of p-spaces with an Application to Convolution Operators, Trans. Amer. Math. Soc. 154 (1971), 69-82

[15] C. Herz and N. Riviere, Estimates for Translation-invariant Operators on Spaces with Mixed Norms, Studia Math. 44 (1972), $511-515$

[16] T. Kawata, Fourier Analysis in Probability Theory, New York 1972

[17] H. KöNIG, On the Complex Grothendieck Constant in the n-dimensional Case, In: Geometry of Banach Spaces (Proc. of Conference held in Strobl, Austria 1989), Cambridge Univ. Press, Cambridge 1990, $181-198$

[18] S. G. Krein, Ju. I. Petunin and E. M. Semenov, Interpolation of Linear Operators, AMS, Providence 1982

[19] J. I. KRIVINE, Théorèmes de Factorisation dans les Espaces Réticulés, Seminaire Maurey-Schwartz 1973-1974, Exposes XXII-XXIII, 22 pp.

[20] J. I. Krivine, Sur la Complexification des Opérateurs de $L^{\infty}$ dans $L^{1}$, C. R. Acad. Sci. Paris 284 (1977), $377-379$

[21] J. I. Krivine, Constantes de Grothendieck et Fonctions de Type Positif sur les Sphères, Adv. Mat. 31 (1979), 16-30

[22] S. Kwapien, Operators Factorizable through $L^{p}$-spaces, Bull. Soc. Math. France Mem. 31-32 (1972), 215-22

[23] P. LeVy, Calcul des Probabilities, Gauthier-Villars, Paris 1924

[24] J. Lindenstrauss and A. PelCZYNSKI, Absolutely Summing Operators in $L_{p}$-spaces and their Applications, Studia Math. 29 (1968), 275-326

[25] J. Lindenstrauss and L. TSafRIRI, Classical Banach Spaces II. Function Spaces, Springer-Verlag 1979

[26] W. Litmann, C. McCarthy and N. Riviere, $L^{p}$-multiplier Theorems, Studia Math. 30 (1968), $193-217$

[27] L. Maligranda and L. E. Persson, On Clarkson's Inequalities and Interpolation, Math. Nachr. 155 (1992), $187-197$

[28] J. MarcinKIEwicz and A. Zygmund, Quelquels Inéqualités pour les Opérations Linéaires, Fund. Math. 32 (1939), 115-121; Reprinted in: J. Marcinkiewicz, Collected Papers, PWN, Warszawa $1964,541-546$

[29] C. McCarthy, A Multiplier Counter-example for Mixed-norm Spaces, Studia Math. 49 (1974), $209-216$

[30] D. M. OnerLin, Translation-invariant Operators of Weak Type, Pacific J. Math. 85 (1979), $155-164$

[31] A. PelczYnsski, Norms of Classical Operators in Function Spaces, Asterisque 13(1985), 137- 162

[32] A. PIETsch, Operator Ideals North-Holland, Amsterdam-Oxford-New York 1980

[33] G. PISIER, Grothendieck's Theorem for Noncommutative $C^{*}$-algebras, with an Appendix on Grothendieck's Constants, J. Functional Anal. 29 (1978), 397-415

[34] G. Pisier, Factorisation of Linear Operators and Geometry of Banach Spaces, CBMS no. 60 , Amer. Math. Soc. 1986, 1-154 
[35] M. Riesz, Sur les Maxima des Formes Bilinéaires et sur les Fonctionelles Linéaires, Acta Math. 49 (1926), 465 - 497; Reprinted in: Marcel Riesz, Collected Papers, Springer-Verlag 1988, 377-409

[36] J. L. Rubio De Francia, Continuity and Pointwise Convergence of Operators in Vector Valued $L^{p}$ Spaces, Boll. Un. Math. Italy 17-B (1980), 650-660

[37] J. L. Rubio DE Francia, Vector Valued Inequalities for Operators in $L^{p}$ Spaces, Bull. London Math. Soc. 12 (1980), 211-215

[38] J. L. Rubio De Francia, Weighted Norm Inequalities and Vector Valued Inequalities, Lecture Notes in Math. 908, 86-101, 1982

[39] J. L. Rubio de Francia and J. L. Torrea, Vector Extensions of Operators in $L^{p}$ Spaces, Pacific J. Math. 105 (1983), $227-235$

[40] Ju. Sokolovski, On the Norm of the Complex Extension of the Linear Operator, Mat. Issled. 54 (1980), 152-154 (in Russian)

[41] S. B. Stechkin, On the Best Lacunary System of Functions, Izv. Acad. Nauk SSSR, Ser. Mat. 25 (1961), 357-366 (in Russian)

[42] J. Szulga, On p-absolutely Summing Operators Acting on Banach Lattices, Studia Math. 81 (1985), 53-63

[43] A. E. TAYLOR, The Norm of a Real Transformation in Minkowski Space, L'Enseign. Math. 4 (1958)

[44] G. O. Thorin, Convexity Theorems Generalizing Those of M. Riesz and Hadamard with some Applications, Comm. Sem. Math. Univ. Lund = Medd. Lunds Univ. Sem. 9 (1948), I -58

[45] A. Tonge, Polarization and the Two-dimensional Grothendieck Inequality, Math. Proc. Camb. Phil. Soc. 95 (1984), 313-318

[46] I. E. VerbickII, Some Relations Between the Norm of an Operator and that of its Complex Extension, Mat. Issled. 42 (1976), 3-12 (Russian)

[47] I. E. VerbickII and P. P. SEREDA, On the Norm of the Complex Extension of Operator, Mat. Issled. 37 (1975), $201-206$ (Russian)

[48] J. H. Welis and L. R. Williams, Imbedding and Extension Problems in Analysis, Springer-Verlag, Berlin 1975

[49] A. Zygmund, Two Notes on Inequalities, J. Math. and Physics 21 (1942), 117-123

[50] A. Zygmund, Trigonometric Series, Cambridge Univ. Press 1959

Departamento de Matematicas

Facultad de Ciencias

Universidad Central de Venezuela

Apartado 20513

Caracas 1020-A

Venezuela
Department of Mathematics

Luleå University

$S$-97187 Luleå

Sweden

e-mail address: lech@sm.luth.se 